\title{
Mean curvature flow with a constant forcing
}

\author{
Zuhan Liu
}

\begin{abstract}
In this paper, the motion of surface by its mean curvature and a forcing term $\theta$ was studied. We show that to each uniformly convex bounded initial surface $M_{0}$, there exists a unique $\theta^{*}$ such that the surface shrinks or expands depending on whether $\theta<\theta^{*}$ or $\theta>\theta^{*}$. Also, we show that the surface with $\theta=\theta^{*}$ converges to a limiting surface.
\end{abstract}

Mathematics Subject Classification. 35K57, 58E15.

Keywords. Mean curvature flow, Viscosity solution, Comparison principle, Interface.

\section{Introduction}

The study of curvature flow has been of great interest recently. One says that a surface moves by mean curvature, if at each point its normal velocity is proportional to the mean curvature. This evolution mode has been named mean curvature motion (or flow).

A more general law is also of interest. In several physical systems, under stationary conditions the mean curvature of a surface is constrained to coincide with some field. As the latter evolves, a natural evolution mode consists in the surface moving with normal velocity proportional to the difference between the mean curvature and the field. This will be referred to as mean curvature flow with a forcing term. For instance, let us consider phase transition in a solid-liquid system $[8,14,25]$. Let us denote by $\theta$ the temperature, by $M_{t}$ the interface between phases, by $v$ its normal velocity, by $H$ the sum of the principal curvatures. Let us assume $H$ positive for a solid ball, $v$ positive for melting and negative for freezing. The mean curvature flow (with a forcing term and with normalized coefficients) then reads

$$
v=H-\theta \quad \text { on } \quad M_{t} \text {. }
$$

Now we briefly describe some mathematical advances concerning this problem. 
Case I $\theta=0$. When the initial surface $M_{0}$ is convex, then as $t \rightarrow T$ the surfaces $M_{t}$ become spherical and contract to a point. $T$ denote a finite critical time at which the curvature of the surface becomes unbounded. This result was obtained by Gage and Hamilton [12] in one-dimensional case and by Huisken [16] in higher dimensional cases; it was then extended by Grayson [13] to arbitrary embedded curves. On the other hand, in higher dimensional cases, nonconvex surfaces may become singular without shrinking to point in the embedded case. Then one can analyze the behavior near a singularity with rescaling techniques $[1-3,17-19,26,28-30]$. In order to describe the behavior of surface $M_{t}$ after singularity happens, Evans and Spruck studied mean curvature flow by viscosity solution theory [10,22]. Almgren, Taylor, Wang, Brakke and Illmanen studied mean curvature flow by geometric measure theory $[4,6,21]$.

Case II $\theta \neq 0$. In one-dimensional case, when the initial curve $M_{0}$ is convex, Chou and Zhu studied existence of global classical solutions to mean curvature flow with a forcing term [9]. Rather complete results are obtained by them. In higher dimensional cases, existence of global weak solutions to this flow was obtained by Chen et al. [7], Luckhaus and Sturzenhecker [23], Soner [27], respectively.

In this paper, using the techniques of Huisken [16], and the viscosity solution method of Evans and Spruck [10], we obtain existence of global classical solutions to (1.1) for uniformly convex initial interface in higher dimensions. Our work borrows many ingredients from $[10,16,27]$. In order to state our theorem, we need to transform (1.1) into partial differential equation. Assume $M_{0}$ is a compact, uniformly convex n-dimensional hypersurface without boundary, which is smoothly imbedded in $\mathbb{R}^{n+1}$, and suppose that $M_{0}$ is represented locally by some diffeomorphism

$$
F_{0}: \quad \mathbb{R}^{n} \supset U \rightarrow F_{0}(U) \subset M_{0} \subset \mathbb{R}^{n+1} .
$$

We transform (1.1) into the following equations

$$
\begin{aligned}
& \frac{\partial}{\partial t} F(p, t)=(-H(x, t)+\theta) \nu(x, t), \quad x \in U, t \geq 0, \\
& F(\cdot, 0)=F_{0}
\end{aligned}
$$

where $H$ is the mean curvature and $\nu$ the outer unit normal, respectively at the point $F(x, t)$ of the surface $M_{t}=F(\cdot, t)(U)$. The signs are chosen such that the mean curvature of a convex surface is positive. We have the following main theorem.

Theorem 1.1. For any given smooth and uniformly convex $M_{0}$, i.e., the eigenvalues of its second fundamental form are strictly positive everywhere, there exists a positive $\theta^{*}$ such that the maximal solution $M_{t}$ of (1.2) starting at $M_{0}$ is uniformly convex for $t \in\left(0, t_{\max }\right)$, and the following hold:

(a) When $0<\theta<\theta^{*}, t_{\max }$ is finite and $M_{t}$ shrinks to a round point as $t \rightarrow t_{\max }$.

(b) When $\theta=\theta^{*}, t_{\max }=+\infty$ and $M_{t}$ converges to a sphere with radius $\frac{n}{\theta^{*}}$. 
(c) When $\theta>\theta^{*}, t_{\max }=+\infty$ and $M_{t}$ expands to infinity as $t$ approaches infinity. Moreover, $\frac{F(\cdot, t)}{t}$ converges smoothly to a sphere with radius $\theta$.

This paper is organized as follows. In Sect. 2, we derive various evolution equations. In Sect. 3, we give a pinching estimate. In Sects. 4, 5, we derive some derivative estimate. In Sect. 6 , we prove the claim(b) of Theorem 1.1. In Sect. 7, we prove the claim(c) of Theorem 1.1.

\section{Evolution equations and convexity properties}

We will use the same notation as in [16]. In particular we write $g=\left\{g_{i j}\right\}$ and $A=\left\{h_{i j}\right\}$ for the metric and the second fundamental form and use the following notations

$$
\begin{gathered}
H=g^{i j} h_{i j}, \quad|A|^{2}=g^{i j} g^{k l} h_{i k} h_{j l}, \\
C=g^{i j} g^{k l} g^{m n} h_{i k} h_{l m} h_{n j}, \quad Z=H C-|A|^{4} .
\end{gathered}
$$

Lemma 2.1. We have the equations

$$
\begin{gathered}
\frac{\partial}{\partial t} g_{i j}=2(\theta-H) h_{i j} \\
\frac{\partial}{\partial t} h_{i j}=\Delta h_{i j}-2 H h_{i m} h_{j}^{m}+\theta h_{i m} h_{j}^{m}+|A|^{2} h_{i j} .
\end{gathered}
$$

Proof. We can derive (2.1), (2.2) by proceeding exactly as in [16], from the basic equation (1.2).

From (2.1), we have

$$
\frac{\partial}{\partial t} g^{i j}=-2(\theta-H) g^{i m} g^{j n} h_{m n}
$$

By contraction we easily obtain from Lemma 2.1 evolution equations for other quantities formed from $g$ and $A$.

Corollary 2.2. We have

$$
\begin{aligned}
& \frac{\partial}{\partial t} H=\Delta H+(H-\theta)|A|^{2}, \\
& \frac{\partial}{\partial t}|A|^{2}=\Delta|A|^{2}-2|\nabla A|^{2}+2|A|^{4}-2 \theta C, \\
& \frac{\partial}{\partial t}\left(|A|^{2}-\frac{1}{n} H^{2}\right)=\Delta\left(|A|^{2}-\frac{1}{n} H^{2}\right)-2\left(|\nabla A|^{2}-\frac{1}{n}|\nabla H|^{2}\right) \\
& \quad+2|A|^{2}\left(|A|^{2}-\frac{1}{n} H^{2}\right)+\frac{2}{n} \theta\left(H|A|^{2}-n C\right), \\
& \frac{\partial}{\partial t} \sqrt{\operatorname{det} g_{i j}}=-H(H-\theta) \sqrt{\operatorname{det} g_{i j}} .
\end{aligned}
$$


Since the initial hypersurface $M_{0}$ is uniformly convex, there is $0<\varepsilon \leq \frac{1}{n}$ such that at $t=0$

$$
h_{i j} \geq \varepsilon H g_{i j}
$$

holds everywhere on $M_{0}$. Whereas the evolution equation for $H$ does not yield an immediate lower bound for the mean curvature, we can show that inequality (2.7) is preserved.

Theorem 2.3. If the initial hypersurface $M_{0}$ is uniformly convex, then $M_{t}$ stays uniformly convex and inequality (2.7) remains true with a uniformly $0<\varepsilon \leq \frac{1}{n}$ for all $t \geq 0$ where the solution of (1.2), (1.3) exists.

Proof. It follows immediately from Lemma 2.1 that uniform convexity is preserved since the absolute terms in the evolution equation for $A$ vanish at null-eigenvectors of $A$. To prove that inequality (2.7) is preserved, we will use Hamilton's maximum principle for tensors [15], and consider

$$
M_{i j}=h_{i j}-\varepsilon H g_{i j} .
$$

From Lemma 2.1 and Corollary 2.2 (i) we obtain

$$
\begin{gathered}
\frac{\partial}{\partial t} M_{i j}=\Delta M_{i j}+N_{i j}, \\
N_{i j}=-2 H h_{i m} h^{m}{ }_{j}+\theta h_{i m} h_{j}^{m}+|A|^{2} h_{i j} \\
-\varepsilon|A|^{2}(H-\theta) g_{i j}-2 \varepsilon H(\theta-H) h_{i j} .
\end{gathered}
$$

We have only to check that $N_{i j}$ is non-negative on the null-eigenvectors of $M_{i j}$ at some $\left(x_{0}, t_{0}\right)$ we may arrange coordinates such that at $\left(x_{0}, t_{0}\right), X=$ $e_{1}, g_{i j}=\delta_{i j}$ and $h_{i j}$ is diagonal. Then

$$
\begin{aligned}
N_{i j} X^{i} X^{j}= & N_{11} \\
= & -2 \varepsilon^{2} H^{3}+\theta \varepsilon^{2} H^{2}+\varepsilon H|A|^{2}-\varepsilon|A|^{2} H \\
& +\varepsilon|A|^{2} \theta-2 \varepsilon^{2} \theta H^{2}+2 \varepsilon^{2} H^{3} \\
= & \varepsilon \theta\left(|A|^{2}-\varepsilon H^{2}\right) .
\end{aligned}
$$

This is non-negative since $\theta>0,0<\varepsilon \leq \frac{1}{n}$ and always $|A|^{2} \geq \frac{1}{n} H^{2}$.

Lemma 2.4. ([16]) If inequality (2.7) holds with $H>0, \varepsilon>0$ at some point of a hypersurface, then we have at that point

$$
\begin{gathered}
\text { (i) } Z \geq n \varepsilon^{2} H^{2}\left(|A|^{2}-\frac{1}{n} H^{2}\right), \\
\text { (ii) }\left|\nabla_{i} h_{k l} H-\nabla_{i} H h_{k l}\right|^{2} \geq \frac{1}{2} \varepsilon^{2} H^{2}|\nabla H|^{2}, \\
\text { (iii) } n C-H|A|^{2} \geq 2 n \varepsilon H\left(|A|^{2}-\frac{1}{n} H^{2}\right) \text {. }
\end{gathered}
$$

\section{A pinching estimate}

Theorem 3.1. There are $\delta>0$ depending only on $M_{0}$, n, and $C_{0}<\infty$ depending only on $M_{0}$, such that 


$$
|A|^{2}-\frac{1}{n} H^{2} \leq C_{0} H^{2-\delta} .
$$

holds for all $0 \leq t<T_{0}<+\infty$ where the solution of problem (1.2)-(1.3) exists.

We want to bound the function

$$
f_{\sigma}=|A|^{2}-\frac{1}{n} H^{2} / H^{2-\sigma}
$$

for some small $\sigma>0$ and begin with an evolution equation for $f_{\sigma}$. First, we prove several lemmas.

Lemma 3.2. We have with $\alpha=2-\sigma$ that

$$
\begin{aligned}
\frac{\partial}{\partial t} f_{\sigma}= & \Delta f_{\sigma}+\frac{2(\alpha-1)}{H}\left\langle\nabla_{l} H, \nabla_{l} f_{\sigma}\right\rangle-\frac{2}{H^{\alpha+2}}\left|\nabla_{l} h_{i j} H-\nabla_{l} H h_{i j}\right|^{2} \\
& -\frac{(2-\alpha)(\alpha-1)}{H^{\alpha+2}}\left(|A|^{2}-\frac{1}{n} H^{2}\right)|\nabla H|^{2}+\frac{\theta}{H^{3-\sigma}}\left(|A|^{4}-H C\right) \\
+\sigma|A|^{2} \frac{(H-\theta)}{H} f_{\sigma}, & \\
\frac{\partial}{\partial t} f_{\sigma} \leq \Delta f_{\sigma} & +\frac{2(\alpha-1)}{H}\left\langle\nabla_{i} H, \nabla_{i} f_{\sigma}\right\rangle-\frac{\varepsilon^{2}}{H^{\alpha}}|\nabla H|^{2}+\sigma|A|^{2} f_{\sigma}-2 \varepsilon^{2} \theta H f_{\sigma} .
\end{aligned}
$$

Proof. By computing as in [16], we have

$$
\begin{aligned}
& \frac{\partial}{\partial t} f_{\sigma}= \frac{1}{H^{\alpha}}\left(\Delta|A|^{2}-2|A|^{4}-\theta C\right)+|A|^{2}\left(-\frac{\alpha H^{\alpha-1}}{H^{2 \alpha}}\right) \cdot\left(\Delta H+(H-\theta)|A|^{2}\right) \\
&-\frac{1}{n}(2-\alpha) H^{1-\alpha} \cdot\left(\Delta H+(H-\theta)|A|^{2}\right) \\
&=\left(H \Delta|A|^{2}-\alpha|A|^{2} \Delta H\right) \frac{1}{H^{\alpha+1}}-\frac{2-\alpha}{n} H^{1-\alpha} \Delta H-\frac{2}{H^{\alpha}}|\nabla A|^{2} \\
&+(2-\alpha)|A|^{2} f_{\sigma}+\theta \frac{|A|^{2}}{H^{1+\alpha}}\left(\alpha|A|^{2}-\frac{2-\alpha}{n} H^{2}\right) \frac{2 \theta}{1+\alpha} C, \\
& \nabla_{i} f_{\sigma}=\left(H \nabla_{i}|A|^{2}-\alpha|A|^{2} \nabla_{i} H\right) / H^{\alpha+1}-\frac{2-\alpha}{n} H^{1-\alpha} \nabla_{i} H, \\
& \Delta f_{\sigma}= \frac{\left(H \Delta|A|^{2}-\alpha|A|^{2} \Delta H\right)}{H^{\alpha+1}}-\frac{2-\alpha}{n} H^{1-\alpha} \Delta H-\frac{2 \alpha}{H^{\alpha+1}}\left\langle\nabla_{i}|A|^{2}, \nabla_{i} H\right\rangle \\
&+\alpha(\alpha+1) \frac{|A|^{2}}{H^{\alpha+2}}|\nabla H|^{2}-\frac{1}{n} \frac{(2-\alpha)(1-\alpha)}{H^{\alpha}}|\nabla H|^{2}, \\
&\left|\nabla_{i} h_{k l} \cdot H-\nabla_{i} H \cdot h_{k l}\right|^{2}=H^{2}|\nabla A|^{2}+|A|^{2}|\nabla H|^{2}-\left\langle\nabla_{i}|A|^{2}, \nabla_{i} H\right\rangle H .
\end{aligned}
$$

The conclusion (3.2) follows. From Lemma 2.4, $\theta>0$, we derive the inequality (3.3). 
Lemma 3.3. There exists a constant $C_{1}$ such that

$$
\left(\int_{M_{t}} f_{\sigma}^{p} d \mu\right)^{\frac{1}{p}} \leq C_{1}
$$

for $p \geq 200 \varepsilon^{-2}, \sigma \leq n \cdot 2^{-4} \varepsilon^{3} p^{-\frac{1}{2}}$.

Proof. Multiplying (3.3) by $p f_{\sigma}^{p-1}$ as in [16], we obtain

$$
\begin{aligned}
& \frac{\partial}{\partial t} \int f_{\sigma}^{p} d \mu+p(p-1) \int f_{\sigma}^{p-2}\left|\nabla f_{\sigma}\right|^{2} d \mu+\varepsilon^{2} p \int \frac{1}{H^{\alpha}} f_{\sigma}^{p-1}|\nabla H|^{2} d \mu \\
& \leq 2(\alpha-1) \int \frac{1}{H} f_{\sigma}^{p-1}|\nabla H|\left|\nabla f_{\sigma}\right| d \mu+\sigma p \int A^{2} f_{\sigma}^{p} d \mu \\
& \quad-2 \varepsilon^{2} p \theta \int H f_{\sigma}^{p} d \mu+\int H(\theta-H) f_{\sigma}^{p} d \mu .
\end{aligned}
$$

Note that $\alpha \leq 2,|A|^{2} \leq H^{2}, a b \leq \frac{\eta}{2} a^{2}+\frac{1}{2 \eta} b^{2}, f_{\sigma} \leq H^{2-\alpha}$. We have

$$
\begin{aligned}
& 2(\alpha-1) p \int \frac{1}{H} f_{\sigma}^{p-1} \cdot|\nabla H| \cdot\left|\nabla f_{\sigma}\right| \\
& \quad \leq \frac{1}{2} p(p-1) \int f_{\sigma}^{p-1}\left|\nabla_{\sigma}\right|^{2} d \mu+\frac{2 p}{p-1} \int f_{\sigma}^{p-1} \cdot \frac{1}{H^{\alpha}} \cdot|\nabla H|^{2} d \mu .
\end{aligned}
$$

Take $p$ satisfying $p-1 \geq 200 \varepsilon^{-2}-1 \geq 100 \varepsilon^{-2}$, we obtain

$$
\begin{aligned}
& \frac{\partial}{\partial t} \int f_{\sigma}^{p} d \mu+\frac{1}{2} p(p-1) \int f_{\sigma}^{p-2}\left|\nabla f_{\sigma}\right|^{2} d \mu+\frac{49}{50} \varepsilon^{2} p \int \frac{1}{H^{\alpha}} f_{\sigma}^{p-1}|\nabla H|^{2} d \mu \\
& \quad \leq \sigma p \int H^{2} f_{\sigma}^{p} d \mu-2 \varepsilon^{2} p \theta \int H f_{\sigma}^{p} d \mu+\int H(\theta-H) f_{\sigma}^{p} d \mu .
\end{aligned}
$$

Now by using Lemma 5.4 in [16] with $\eta=\varepsilon p^{-\frac{1}{2}} / 4$, we derive for all $p \geq$ $200 \varepsilon^{-} 2, \sigma \leq n \cdot 2^{-4} \cdot \varepsilon^{3} p^{-\frac{1}{2}}$

$$
\frac{\partial}{\partial t} \int f_{\sigma}^{p} d \mu \leq 0
$$

Hence

$$
\left(\int f_{\sigma}^{p} d \mu\right)^{\frac{1}{p}} \leq C_{1}
$$

The proof of Theorem 3.1. By the method used by Huisken [16], we let $f_{\sigma, k}=$ $\max \left(f_{\sigma}-k, 0\right)$ and $A(k)=\left\{x \in M: f_{\sigma}>k\right\}$. Multiplying (3.3) with $p f_{\sigma, k}^{p-1}$, we derive for $p \varepsilon^{2}$ large

$$
\begin{aligned}
& \frac{\partial}{\partial t} \int f_{\sigma, k}^{p} d \mu+\frac{1}{2} p(p-1) \int_{A(k)}\left|\nabla f_{\sigma}\right|^{2} f_{\sigma, k}^{p-2} d \mu \\
& \quad \leq \sigma p \int_{A(k)} H^{2} f_{\sigma, k}^{p-1} f_{\sigma} d \mu-\varepsilon^{2} p \int_{A(k)} \theta H f_{\sigma, k}^{p-1} f_{\sigma} d \mu-\int_{A(k)} H^{2} f_{\sigma, k}^{p-1} d \mu .
\end{aligned}
$$


Proceeding exactly as in [16], we see that there are a fixed finite $p_{0}$ and a $\sigma_{0}>0$ such that for all $0<\sigma \leq \sigma_{0}, 0<T_{0}<\infty$ and $k \geq k_{1}>0$

$$
f_{\sigma} \leq k+d, d^{p_{0}}=C_{2} 2^{p_{0} \gamma / \gamma+1}\|A(k)\|_{T_{0}}^{\gamma-1}
$$

where $k_{1}$ and $C_{2}$ are constants depending only on $n, \varepsilon, C_{1}, M_{0}$ and

$$
\|A(k)\|_{T_{0}}=\int_{0}^{T_{0}} \int_{A(k)} d \mu d t .
$$

To show that this term is bounded independently of $T$ we show that $\|A(k)\|=$ $\int_{A(k)} d \mu$ decays exponentially for some $k \geq k_{1}$.

Choose a fixed $p$ such that (3.5) holds and then $\sigma \leq \frac{1}{2} p^{-1}$, such that

$$
\begin{aligned}
\frac{\partial}{\partial t} \int_{A\left(k_{1}\right)} f_{\sigma, k_{1}}^{p} d \mu & \leq-\frac{1}{2} \int_{A\left(k_{1}\right)} H^{2} f_{\sigma, k_{1}}^{p-1} f_{\sigma} d \mu \\
& \leq-\frac{1}{2} \int_{A\left(k_{1}\right)} H^{2} f_{\sigma, k_{1}}^{p} d \mu
\end{aligned}
$$

since $f_{\sigma} \geq f_{\sigma, k_{1}}$. Moreover, on $A\left(k_{1}\right)$ we have

$$
k_{1} \leq \frac{|A|^{2}-\frac{1}{n} H^{2}}{H^{2-\sigma}} \leq H^{\sigma}
$$

and therefore

$$
\frac{\partial}{\partial t} \int_{A\left(k_{1}\right)} f_{\sigma, k_{1}}^{p} d \mu \leq-\frac{1}{2} k_{1}^{2 / \sigma} \int_{A\left(k_{1}\right)} f_{\sigma, k_{1}}^{p} d \mu
$$

so we get

$$
\int_{A\left(k_{1}\right)} f_{\sigma, k_{1}}^{p} d \mu \leq\left. e^{-\delta t} \int_{A\left(k_{1}\right)} f_{\sigma, k_{1}}^{p} d \mu\right|_{t=0},
$$

where $\delta=\frac{1}{2} k_{1}^{2 / \sigma}$. Then let $k=k_{1}+1$ and observe that $1 \leq\left(f_{\sigma}-k_{1}\right)^{p}$ on $A\left(k_{1}+1\right)$. Thus we have

$$
\begin{aligned}
\left|A\left(k_{1}+1\right)\right| & =\int_{A\left(k_{1}+1\right)} d \mu \leq \int_{A\left(k_{1}+1\right)}\left(f_{\sigma}-k_{1}\right)^{p} d \mu \\
& \leq \int_{A\left(k_{1}\right)}\left(f_{\sigma}-k_{1}\right)^{p} d \mu=\int_{A\left(k_{1}\right)} f_{\sigma, k_{1}}^{p} d \mu \leq C e^{-\delta t} .
\end{aligned}
$$

Then the proof of Theorem 3.1 is completed.

\section{Gradient estimate for the mean curvature}

Theorem 4.1. For all $\eta>0$ there is a constant $C_{3}<\infty$ depending only on $\eta$, $M_{0}$ and $\theta$ such that

$$
|\nabla H|^{2} \leq \eta H^{4}+C_{3}
$$

holds on $M_{t}$ for all $0 \leq t \leq T$.

In order to prove this theorem, we will use the method used by Huisken [16] and need the following lemmas. 
Lemma 4.2. We have

(i) $\frac{\partial}{\partial t}|\nabla H|^{2}=\Delta|\nabla H|^{2}-2\left|\nabla^{2} H\right|^{2}+2|A|^{2}|\nabla H|^{2}+2\left\langle\nabla_{i} H \cdot h_{m j}, \nabla_{j} H \cdot h_{i m}\right\rangle$ $+2(H-\theta)\left\langle\nabla_{i}|A|^{2}, \nabla_{j} H\right\rangle-2 \theta\left\langle h_{i j}, \nabla_{i} H \cdot \nabla_{j} H\right\rangle$,

(ii) $\frac{\partial}{\partial t}|\nabla H|^{2} \leq \Delta|\nabla H|^{2}+8 n H(H+\theta)|\nabla A|^{2}$.

Proof. (i) By computing as in [16]

$$
\begin{aligned}
& \frac{\partial}{\partial t}|\nabla H|^{2}=\frac{\partial}{\partial t}\left(g^{i j} \nabla_{i} H \cdot \nabla_{j} H\right) \\
&=2\left\langle\nabla_{i} H, \nabla_{i}\left(\Delta H+(H-\theta)|A|^{2}\right)\right\rangle+2(H-\theta)\left\langle h_{i j}, \nabla_{i} H \nabla_{j} H\right\rangle, \\
& \Delta|\nabla H|^{2}=2 g^{k l} \Delta\left(\nabla_{k} H\right) \nabla_{l} H+2\left|\nabla^{2} H\right|, \\
& \Delta\left(\nabla_{k} H\right)=\nabla_{k}(\Delta H)+g^{i j} \nabla_{i} H\left(H h_{k j}-h_{k m} g^{m n} h_{m j}\right), \\
& 2 g^{i j} \nabla_{i}(\Delta H) \nabla_{j} H=2 g^{i j} \Delta\left(\nabla_{i} H\right) \nabla_{j} H-2 g^{i j}\left(g^{s p} \nabla_{s} H\left(H h_{i p}-h_{i m} g^{m n} h_{n p}\right)\right) \nabla_{j} H \\
&=\Delta|\nabla H|^{2}-2\left|\nabla^{2} H\right|^{2}-2 g^{i j} g^{s p} \nabla_{s} H \cdot H h_{i p} \cdot \nabla_{j} H \\
& \quad+2 g^{i j} g^{s p} \nabla_{s} H \cdot h_{i m} g^{m n} h_{n p} \cdot \nabla_{j} H \\
&=\Delta|\nabla H|^{2}-2\left|\nabla^{2} H\right|^{2}-2 H\left\langle h_{i j}, \nabla_{i} H \cdot \nabla_{j} H\right\rangle \\
&+2\left\langle\nabla_{i} H \cdot h_{m j}, \nabla_{j} H \cdot h_{i m}\right\rangle,
\end{aligned}
$$

hence conclusion (i) follows.

(ii) Note that

$$
\begin{gathered}
\left|2(H-\theta)\left\langle\nabla_{i} H, \nabla_{i}|A|^{2}\right\rangle\right| \\
=2\left|(H-\theta)\left\langle\nabla_{i} H, \nabla_{i}\left(h_{k l} h^{k l}\right)\right\rangle\right| \\
\leq 4 n H^{2}|\nabla A|^{2}+4 n \theta H|\nabla A|^{2}, \\
h_{i j} \geq 0, \quad|\nabla H|^{2} \leq n|\nabla A|^{2} .
\end{gathered}
$$

Using Hölder inequality, we obtain

$$
\frac{\partial}{\partial t}|\nabla H|^{2} \leq \Delta|\nabla H|^{2}+8 n H(H+\theta)|\nabla A|^{2} .
$$

Then conclusion (ii) follows.

Lemma 4.3. For all $0 \leq t \leq T$, we have the estimate

$$
\begin{aligned}
& \text { (i) } \frac{\partial}{\partial t}\left(|A|^{2}-\frac{1}{n} H^{2}\right) \leq \Delta\left(|A|^{2}-\frac{1}{n} H^{2}\right)-\frac{2(n-1)}{3 n}|\nabla A|^{2}+2|A|^{2}\left(|A|^{2}-\frac{1}{n} H^{2}\right) \text {, } \\
& \text { (ii) } \frac{\partial}{\partial t}\left(H^{2}\left(|A|^{2}-\frac{1}{n} H^{2}\right)\right) \leq \Delta\left(H^{2}\left(|A|^{2}-\frac{1}{n} H^{2}\right)\right)-\frac{2(n-1)}{3 n} H^{2}|\nabla A|^{2} \\
& +4 H^{2}|A|^{2}\left(|A|^{2}-\frac{1}{n} H^{2}\right)-4 H\left\langle\nabla_{i} H, \nabla_{i}\left(|A|^{2}-\frac{1}{n} H^{2}\right)\right\rangle, \\
& \text { (iii) } \frac{\partial}{\partial t}\left(H\left(|A|^{2}-\frac{1}{n} H^{2}\right)\right) \leq \Delta\left(H\left(|A|^{2}-\frac{1}{n} H^{2}\right)\right)-\frac{2(n-1)}{3 n} H|\nabla A|^{2} \\
& +3 H|A|^{2}\left(|A|^{2}-\frac{1}{n} H^{2}\right)-2\left\langle\nabla_{i} H, \nabla_{i}\left(|A|^{2}-\frac{1}{n} H^{2}\right)\right\rangle .
\end{aligned}
$$


Proof. Claim (i), (ii) follow from Lemma 3.4 (i) in [16]. So we only need to prove claim (iii). By computing as in [16], (2.3) and (2.5), we have

$$
\begin{aligned}
\frac{\partial}{\partial t}( & \left.\left(|A|^{2}-\frac{1}{n} H^{2}\right) H\right) \\
= & \left(|A|^{2}-\frac{1}{n} H^{2}\right)\left(\Delta H+(H-\theta)|A|^{2}\right)+H\left[\Delta\left(|A|^{2}-\frac{1}{n} H^{2}\right)\right. \\
& \left.-2\left(|\nabla A|^{2}-\frac{1}{n}|\nabla H|^{2}\right)+2|A|^{2}\left(|A|^{2}-\frac{1}{n} H^{2}\right)-2 \theta\left(C-\frac{1}{n} H|A|^{2}\right)\right] \\
= & \Delta\left(\left(|A|^{2}-\frac{1}{n} H^{2}\right) H\right)-2\left\langle\nabla_{i} H, \nabla_{i}\left(|A|^{2}-\frac{1}{n} H^{2}\right)\right\rangle \\
& +(3 H-\theta)|A|^{2}\left(|A|^{2}-\frac{1}{n} H^{2}\right)-2 H\left(|\nabla A|^{2}-\frac{1}{n}|\nabla H|^{2}\right) \\
& -2 \theta H\left(C-\frac{1}{n} H|A|^{2}\right) \cdot
\end{aligned}
$$

From Lemma 2.2 (ii) in [16], we know the inequality

$$
|\nabla A|^{2}-\frac{1}{n}|\nabla H|^{2} \geq \frac{2(n-1)}{3 n}|\nabla A|^{2} .
$$

Combining (4.2) with (4.3), we have

$$
\begin{array}{r}
\frac{\partial}{\partial t}\left(\left(|A|^{2}-\frac{1}{n} H^{2}\right) H\right) \leq \Delta\left(\left(|A|^{2}-\frac{1}{n} H^{2}\right) H\right)-\frac{2(n-1)}{3 n} H|\nabla A|^{2} \\
+3 H|A|^{2}\left(|A|^{2}-\frac{1}{n} H^{2}\right)-2\left\langle\nabla_{i} H, \nabla_{i}\left(|A|^{2}-\frac{1}{n} H^{2}\right)\right\rangle .
\end{array}
$$

Now we are in a position to prove Theorem 4.1.

Proof. Let $h_{i j}^{0}=h_{i j}-\frac{1}{n} g_{i j}$, using Theorem 3.1, we have

$$
\begin{aligned}
& H\left|\left\langle\nabla_{i} H, \nabla_{i}\left(|A|^{2}-\frac{1}{n} H^{2}\right)\right\rangle\right|=H\left|\left\langle\nabla_{i} H, \nabla_{i}\left(h_{k l}^{0} h^{0 k l}\right)\right\rangle\right| \\
& \quad \leq 2 H|\nabla H|\left|h_{i j}^{0}\right|\left|\nabla_{k} h_{i j}^{0}\right| \leq 2 n C_{0}^{\frac{1}{2}}|\nabla A|^{2} H^{2-\frac{\delta}{2}} \\
& \quad \leq \frac{(n-1)}{3 n} \frac{1}{4} H^{2}|\nabla A|^{2}+C_{4}\left(C_{0}, n, \delta\right)|\nabla A|^{2},
\end{aligned}
$$

and similarly

$$
\theta\left|\left\langle\nabla_{i} H, \nabla_{i}\left(|A|^{2}-\frac{1}{n} H^{2}\right)\right\rangle\right| \leq \frac{n-1}{3 n} \frac{1}{4} \theta H|\nabla A|^{2}+C_{5}\left(C_{0}, n, \delta\right) \theta|\nabla A|^{2} .
$$

Let

$$
g_{1}=(H+\theta) H\left(|A|^{2}-\frac{1}{n} H^{2}\right), \quad g_{2}=(1+\theta)\left(|A|^{2}-\frac{1}{n} H^{2}\right),
$$

then 


$$
\begin{aligned}
\frac{\partial}{\partial t} g_{1} \leq & \Delta g_{1}-\frac{(n-1)}{3 n}(H+\theta) H|\nabla A|^{2}+\left(C_{4}+\theta C_{5}\right)|\nabla A|^{2} \\
& +4 H^{2}|A|^{2}\left(|A|^{2}-\frac{1}{n} H^{2}\right)+3 \theta H|A|^{2}\left(|A|^{2}-\frac{1}{n} H^{2}\right), \\
\frac{\partial}{\partial t} g_{2} \leq & \Delta g_{2}-\frac{2(n-1)}{3 n}(1+\theta)|\nabla A|^{2}+2(1+\theta)|A|^{2}\left(|A|^{2}-\frac{1}{n} H^{2}\right) .
\end{aligned}
$$

Let $N_{1}$ be so large that $N_{1} \cdot \frac{2(n-1)}{3 n} \geq 2 \max \left(C_{4}, C_{5}\right)$, then

$$
\begin{aligned}
& \frac{\partial}{\partial t}\left(g_{1}+N_{1} g_{2}\right) \leq \Delta\left(g_{1}+N_{1} g_{2}\right)-\frac{(n-1)}{3 n}(H+\theta) H|\nabla A|^{2} \\
& \quad-N_{1} \cdot \frac{(n-1)}{3 n}(1+\theta)|\nabla A|^{2} \\
& \quad+\left[4(H+\theta) H|A|^{2}+2 N_{1}(1+\theta)|A|^{2}\right] \cdot\left(|A|^{2}-\frac{1}{n} H^{2}\right) .
\end{aligned}
$$

Now let $N_{2} \cdot \frac{(n-1)}{3 n} \geq 16 n$, then we see from Lemma 4.2 (ii) that the function

$$
g_{3}=|\nabla H|^{2}+N_{2} g_{1}+N_{2} N_{1} g_{2}
$$

satisfies the inequality

$$
\begin{aligned}
\frac{\partial}{\partial t} g_{3} \leq & \Delta g_{3}-N_{2} \frac{n-1}{6 n}(H+\theta) H|\nabla A|^{2}-N_{1} N_{2} \frac{(n-1)}{3 n}(1+\theta)|\nabla A|^{2} \\
& +N_{2}\left[4(H+\theta) H|A|^{2}+2 N_{1}(1+\theta)|A|^{2}\right]\left(|A|^{2}-\frac{1}{n} H^{2}\right) .
\end{aligned}
$$

From Theorem 3.1, we have

$$
\begin{aligned}
& \left.N_{2}\left[4(H+\theta) H|A|^{2}+2 N_{1}(1+\theta)|A|^{2}\right)\right]\left(|A|^{2}-\frac{1}{n} H^{2}\right) \\
& \quad \leq N_{2}\left[4 H^{2}+4 \theta H+2 N_{1}(1+\theta)\right] H^{2} C_{0} \cdot H^{2-\delta} \\
& \quad \leq \eta H^{2} H^{4}+C_{6} H^{2}+C_{7} H^{3}
\end{aligned}
$$

with $C_{6}, C_{7}$ depending only on $\delta, N_{1}, N_{2}, C_{0}, \theta$.

Thus we derive

$$
\begin{aligned}
\frac{\partial}{\partial t} g_{3} \leq & \Delta g_{3}-N_{2} \frac{n-1}{6 n^{2}}(H+\theta) H|\nabla A|^{2}-N_{1} N_{2} \frac{n-1}{3 n^{2}}(1+\theta)|\nabla A|^{2} \\
& +\eta H^{2} H^{4}+C_{8} H^{3} .
\end{aligned}
$$

We want to show now that for any $\tilde{\eta}>0$, there is $C_{9}(\tilde{\eta})$ such that

$$
g_{3}<\tilde{\eta} H^{4}+C_{9} \text { for } \quad 0 \leq t \leq T .
$$

Choose $C_{9}$ so large that this inequality holds at $t=0$ and then suppose there is a first time $t=t_{0} \leq T$ where $g_{3}=\tilde{\eta} H^{4}+C_{9}$ at $x_{0}$. At this point we have $\Delta g_{3} \leq 0, \frac{\partial}{\partial t} g_{3} \geq 0$ and therefore

$$
\begin{aligned}
0 \leq & -\left[N_{2}(H+\theta) H+2 N_{2} N_{1}(1+\theta)\right] \frac{n-1}{6 n^{2}} \cdot\left[\tilde{\eta} H^{4}+C_{9}\right. \\
& \left.-N_{2}\left(H^{2}+\theta H+N_{1}(1+\theta)\right)\left(|A|^{2}-\frac{1}{n} H^{2}\right)\right]+\eta H^{2} H^{4}+C_{9} H^{3} .
\end{aligned}
$$


Using Theorem 3.1 as before we have

$$
\tilde{\eta} H^{4}+C_{9}-N_{2}\left\{\left(H^{2}+\theta H+N_{1}(1+\theta)\right)\left(|A|^{2}-\frac{1}{n} H^{2}\right) \geq \frac{1}{2} \tilde{\eta} H^{4}+\frac{1}{2} C_{9}\right.
$$

provided $C_{9}=C_{9}\left(\tilde{\eta}, \delta, N_{1}, N_{2}, C_{0}, M_{0}\right)$ is large enough. So we get

$$
\begin{aligned}
0 \leq & -\tilde{\eta} \frac{n-1}{12 n^{2}} N_{2} H^{2} H^{4}-N_{2} N_{1} \frac{n-1}{6 n^{2}} C_{9}-N_{2} N_{1} \frac{n-1}{6 n^{2}} \tilde{\eta} H^{4}+\eta H^{2} H^{4} \\
& +C_{9}(\eta) H^{3}
\end{aligned}
$$

and we yield a contradiction if we first choose $\eta \leq \tilde{\eta} \frac{n-1}{12 n^{2}} N_{2}$ and then $C_{9}=$ $C_{9}\left(C_{\delta}, \tilde{\eta}\right)$ large. This completes the proof of Theorem 4.1 .

\section{Higher derivatives estimate}

Let $S * T$ denote any linear combination of tensors formed by contraction with $g$ from $S$ and $T, \nabla^{m} T$ denote $m$-th covariant derivative of tensor $T$.

Theorem 5.1. We have

$$
\begin{aligned}
\frac{\partial}{\partial t}\left|\nabla^{m} A\right|^{2}= & \Delta\left|\nabla^{m} A\right|^{2}-2\left|\nabla^{m+1} A\right|^{2}+\sum_{i+j+k=m} \nabla^{i} A * \nabla^{j} A * \nabla^{k} A * \nabla^{m} A \\
& +\theta \sum_{i+j=m} \nabla^{i} A * \nabla^{j} A * \nabla^{m} A .
\end{aligned}
$$

In order to prove Theorem 5.1, we will use the method used by Huisken [16] and need the following lemmas.

Lemma 5.2. Assume $\bar{A}$ and $B$ are two tensors, and $\frac{\partial}{\partial t} \bar{A}=\Delta \bar{A}+B$. Then

$$
\frac{\partial}{\partial t} \nabla \bar{A}=\Delta \nabla \bar{A}+\nabla R_{m} * \bar{A}+R_{m} * \nabla \bar{A}+\bar{A} * A * \nabla A+\theta \bar{A} * \nabla A+\nabla B
$$

where $R_{m}=\left\{R_{i j k l}\right\}$.

Proof. By computing as in [16]

$$
\begin{aligned}
\frac{\partial}{\partial t} \Gamma_{j k}^{i} & =\frac{1}{2} g^{i l}\left\{\nabla_{j}\left(\frac{\partial}{\partial t} g_{k l}\right)+\nabla_{k}\left(\frac{\partial}{\partial t} g_{j l}\right)-\nabla_{l}\left(\frac{\partial}{\partial t} g_{j k}\right)\right\} \\
& =-g^{i l}\left\{\nabla_{j}\left((H-\theta) h_{k l}\right)+\nabla_{k}\left((H-\theta) h_{j l}\right)-\nabla_{l}\left((H-\theta) h_{j k}\right)\right\} \\
& =A * \nabla A+\theta\left(g^{i l} \nabla_{j} h_{k l}+g^{j l} \nabla_{k} h_{j l}-g^{i l} \nabla_{l} h_{j k}\right) .
\end{aligned}
$$

One has

$$
\frac{\partial}{\partial t} \nabla \bar{A}=\nabla \frac{\partial \bar{A}}{\partial t}+\bar{A} * A * \nabla A+\theta \bar{A} * \nabla A .
$$

Again, one has

$$
\Delta \nabla \bar{A}=\Delta \nabla \bar{A}+\nabla R_{m} * \bar{A}+R_{m} * \nabla \bar{A} .
$$

Hence, the conclusion follows. 
Lemma 5.3. Suppose $A=\left\{h_{i j}\right\}$, then

$\frac{\partial}{\partial t} \nabla^{m} A=\Delta\left(\nabla^{m} A\right)+\sum_{i+j+k=m} \nabla^{i} A * \nabla^{j} A * \nabla^{k} A+\theta \sum_{i+j=m} \nabla^{i} A * \nabla^{j} A$.

Proof. For $m=0$, the conclusion follows from Lemma 2.1. Assume that (5.2) holds for $m$. By Lemma 5.2, we have

$$
\begin{aligned}
\frac{\partial}{\partial t} \nabla^{m+1} A= & \Delta \nabla^{m+1} A+\nabla R_{m} * \nabla^{m} A+R_{m} * \nabla^{m+1} A \\
& +\nabla\left(\sum_{i+j+k=m} \nabla^{i} A * \nabla^{j} A * \nabla^{k} A+\sum_{i+j=m} \nabla^{i} A * \nabla^{j} A\right) \\
= & \Delta \nabla^{m+1} A+\sum_{i+j+k=m+1} \nabla^{i} A * \nabla^{j} A * \nabla^{k} A \\
& +\theta \sum_{i+j=m+1} \nabla^{i} A * \nabla^{j} A,
\end{aligned}
$$

i.e. (5.2) is true for $m+1$. Hence the proof of Lemma 5.3 is completed.

The proof of Theorem 5.1. By computing as in [16]

$$
\begin{aligned}
\frac{\partial}{\partial t}\left|\nabla^{m} A\right|^{2}= & 2\left\langle\nabla^{m} A, \frac{\partial}{\partial t} \nabla^{m} A\right\rangle+A * A * \nabla^{m} A * \nabla^{m} A+\theta A * \nabla^{m} A * \nabla^{m} A \\
= & 2\left\langle\nabla^{m} A, \Delta \nabla^{m} A\right\rangle+2\left\langle\nabla^{m} A, \sum_{i+j+k=m} \nabla^{i} A * \nabla^{j} * \nabla^{k} A\right\rangle \\
& +\left\langle\nabla^{m} A, \theta \sum_{i+j=m} \nabla^{i} A * \nabla^{j} A\right\rangle+A * A * \nabla^{m} A * \nabla^{m} A \\
& +\theta A * \nabla^{m} A * \nabla^{m} A \\
= & 2\left\langle\nabla^{m} A, \Delta \nabla^{m} A\right\rangle+\sum_{i+j+k=m} \nabla^{i} A * \nabla^{j} A * \nabla^{k} A * \nabla^{m} A \\
& +\theta \sum_{i+j=m} \nabla^{i} A * \nabla^{j} A * \nabla^{m} A, \\
& \Delta\left|\nabla^{m} A\right|^{2}=2\left\langle\nabla^{m} A, \Delta \nabla^{m} A\right\rangle+2\left|\nabla^{m+1} A\right|^{2} .
\end{aligned}
$$

We obtain

$$
\begin{aligned}
\frac{\partial}{\partial t}\left|\nabla^{m} A\right|^{2}= & \Delta\left|\nabla^{m} A\right|^{2}-2\left|\nabla^{m+1} A\right|^{2}+\sum_{i+j+k=m} \nabla^{i} A * \nabla^{j} A * \nabla^{k} A * \nabla^{m} A \\
& +\theta \sum_{i+j=m} \nabla^{i} A * \nabla^{j} A * \nabla^{m} A .
\end{aligned}
$$

The proof of Theorem 5.1 is completed. 
Theorem 5.4. We have

$$
\begin{aligned}
& \frac{d}{d t} \int\left|\nabla^{m} A\right|^{2} d \mu+2 \int\left|\nabla^{m+1} A\right|^{2} d \mu \\
& \quad \leq C(n, m)\left(\max _{M_{t}}|A|^{2}+\theta \max _{M_{t}}|A|\right) \int\left|\nabla^{m} A\right|^{2} d \mu .
\end{aligned}
$$

Proof. By computing as in [16]

$$
\begin{aligned}
& \int \frac{\partial}{\partial t}\left|\nabla^{m} A\right|^{2}+2 \int\left|\nabla^{m+1} A\right|^{2} \\
& =\int \sum_{i+j+k=m} \nabla^{i} A * \nabla^{j} A * \nabla^{k} A * \nabla^{m} A+\theta \sum_{i+j=m} \nabla^{i} A * \nabla^{j} A * \nabla^{m} A,
\end{aligned}
$$

we obtain

$$
\begin{aligned}
\frac{\partial}{\partial t} \int\left|\nabla^{m} A\right|^{2}+2 \int\left|\nabla^{m+1} A\right|^{2} \\
\leq N\left\{\int\left|\nabla^{i} A\right|^{2 m / j} \mid\right\}^{j / 2 m}\left\{\int\left|\nabla^{j} A\right|^{2 m / k}\right\}^{k / 2 m}\left\{\int\left|\nabla^{k} A\right|^{2 m / k}\right\}^{k / 2 m} \\
\times\left\{\int\left|\nabla^{m} A\right|^{2}\right\}^{1 / 2}+N \theta\left\{\int\left|\nabla^{i} A\right|^{2 m / i}\right\}^{i / 2 m} \\
\times\left\{\int\left|\nabla^{j} A\right|^{2 m / j}\right\}^{j / 2 m}\left\{\int\left|\nabla^{m} A\right|^{2}\right\}^{1 / 2} .
\end{aligned}
$$

Using the following inequality in [15]

$$
\int_{M}\left|\nabla^{i} A\right|^{2 m / i} d \mu \leq C(m, n) \max |A|^{2(m / i-1)} \int_{M}\left|\nabla^{m} A\right|^{2} d \mu,
$$

we obtain the conclusion.

From Theorem 5.4 and the proof of Lemma 8.3 in [16], we have

Corollary 5.5. If $\max _{M_{t}}|A|^{2} \leq N, \forall 0 \leq t<T<+\infty$, then $\left|\nabla^{m} A\right| \leq N_{m}$ for all $m$. The constant $C_{m}$ depends on $n, M_{0}$ and $N$.

Theorem 5.6. The maximal solution of problem (1.2)-(1.3) with uniformly convex initial hypersurface either exists for all time or shrinks to a point in finite time. Moreover, if $t_{\max }<\infty$, then we have

$$
\max _{M_{t}}|A|^{2} \rightarrow+\infty \text { as } t \rightarrow t_{\max } .
$$

Proof. By the method used by Evans and Spruck [11], we have that the equation (1.2), (1.3) has a unique solution $M_{t}$ for a short time with any smooth compact initial surface $M_{0}$ at $t=0$.

Let $0 \leq t<t_{\max }$ be the maximal time interval where the solution exists. If $t_{\max }<\infty$, by the proof of Theorem 8.1 in [16], we have

$$
\max _{M_{t}}|A|^{2} \rightarrow+\infty \text { as } t \rightarrow t_{\max }
$$

From Theorem 3.1, Theorem 5.1, Corollary 5.5, (5.4), and the proof of Theorem 8.1 in [16, section 8$]$, one yields the conclusion. 


\section{Convergence to a stationary solution}

In this section, we show that to each uniformly convex without boundary initial surface $M_{0}$, there exists a unique $\theta^{*}$ such that the solution of problem (1.2)-(1.3) shrinks or expands depending on whether $\theta<\theta^{*}$ or $\theta>\theta^{*}$. Also, we show that the solution of problem (1.2)-(1.3) with $\theta=\theta^{*}$ converges to a limiting surface.

We begin by stating a comparison principle that is a direct consequence of the comparison principle for viscosity solutions of our mean curvature evolution PDE problem (1.2)-(1.3) [7,10].

Proposition 6.1. Let $M_{t}^{1}$ and $M_{t}^{2}$ be a solution of problem (1.2)-(1.3) with uniformly convex initial surface $M_{0}^{1}, M_{0}^{2}$, respectively. Let $U_{t}^{1}, U_{t}^{2}$ be the domain bounded by $M_{t}^{1}, M_{t}^{2}$, respectively. Suppose $U_{0}^{1} \subset U_{0}^{2}$, then $U_{t}^{1} \subset U_{t}^{2}$ for $t>0$.

Assume $M_{0}$ is a compact, uniformly convex n-dimensional hypersurface without boundary, which is smoothly imbedded in $\mathbb{R}^{n+1}$, and suppose that $M_{0}$ is represented locally by some diffeomorphism

$$
F_{0}: \mathbb{R}^{n} \supset U \rightarrow F_{0}(U) \subset M_{0} \subset \mathbb{R}^{n+1} .
$$

Consider the following equations

$$
\begin{gathered}
\begin{cases}F_{t}=(-H+\theta) \vec{\nu}, & x \in U, t>0, \\
F(\cdot, 0)=F_{0}, & x \in U,\end{cases} \\
\begin{cases}F_{t}^{1}=\left(-H^{1}+\theta^{1}\right) \overrightarrow{\nu^{1}}, & x \in U, t>0, \\
F^{1}(\cdot, 0)=F_{0}, & x \in U\end{cases}
\end{gathered}
$$

where $H, H^{1}$ is mean curvature of the surface $M_{t}, M_{t}^{1}$, respectively.

Proposition 6.2. Assume that $M_{0}$ is a compact, uniformly convex n-dimensional hypersurface without boundary. Suppose that $\left\{M_{t}\right\}_{t>0}$ is the solution of $(6.1)_{\theta}-(6.2),\left\{M_{t}^{1}\right\}_{t>0}$ is the solution of $(6.3)_{\theta^{1}}-(6.4)$. Let $U_{t}$ and $U_{t}^{1}$ is domain bounded by $M_{t}, M_{t}^{1}$, respectively. Suppose that $\theta<\theta^{1}$. Then $U_{t} \subset U_{t}^{1}$ for $t>0$.

In order to prove Proposition 6.2, we will use the viscosity solution theory as in $[7,10]$. Consider the following problem

$$
\begin{cases}u_{t}=\frac{1}{n}\left(\delta_{i j}-\frac{u_{x_{i}} u_{x_{j}}}{|\nabla u|^{2}}\right) u_{x_{i} x_{j}}-\theta|\nabla u|, & (x, t) \in \mathbb{R}^{n+1} \times(0, T) \\ \left.u\right|_{t=0}=g, & x \in \mathbb{R}^{n+1}\end{cases}
$$

where $\nabla u=\left(u_{x_{1}}, u_{x_{2}}, \ldots, u_{x_{n+1}}\right)$.

Definition 6.1. A function $u \in C\left(\mathbb{R}^{n+1} \times[0, T)\right) \bigcap L^{\infty}\left(\mathbb{R}^{n+1} \times(0, T)\right)$ is a weak subsolution of equation (6.5) provided that if $u-\phi$ has a local maximum at a point $\left(x_{0}, t_{0}\right) \in \mathbb{R}^{n+1} \times(0, T)$ for each $\phi \in C^{2}\left(\mathbb{R}^{n+1}\right) \times(0, T)$, then

$$
\left\{\begin{array}{c}
\phi_{t} \leq \frac{1}{n}\left(\delta_{i j}-\phi_{x_{i}} \phi_{x_{j}} /|\nabla \phi|^{2}\right) \phi_{x_{i} x_{j}}-\theta|\nabla \phi| \quad \text { at }\left(x_{0}, t_{0}\right) \\
\text { if } \nabla \phi\left(x_{0}, t_{0}\right) \neq 0
\end{array}\right.
$$


and

$$
\left\{\begin{array}{c}
\phi_{t} \leq \frac{1}{n}\left(\delta_{i j}-\eta_{i} \eta_{j}\right) \phi_{x_{i} x_{j}} \text { at }\left(x_{0}, t_{0}\right) \\
\text { for some } \eta \in \mathbb{R}^{n+1} \text { with }|\eta| \leq 1, \quad \text { if } \nabla \phi\left(x_{0}, t_{0}\right)=0 .
\end{array}\right.
$$

Definition 6.2. A function $u \in C\left(\mathbb{R}^{n+1} \times[0, T)\right)$ is a weak supersolution of equation (6.5) provided that if $u-\phi$ has a local minimum at a point $\left(x_{0}, t_{0}\right) \in$ $\mathbb{R}^{n+1} \times(0, T)$ for each $\phi \in C^{2}\left(\mathbb{R}^{n+1} \times(0, T)\right)$, then

$$
\left\{\begin{array}{l}
\phi_{t} \geq \frac{1}{n}\left(\delta_{i j}-\frac{\phi_{x_{i}} \phi_{x_{j}}}{|\nabla \phi|^{2}}\right) \phi_{x_{i} x_{j}}-\theta|\nabla \phi| \quad \text { at }\left(x_{0}, t_{0}\right) \\
\text { if } \quad \nabla \phi\left(x_{0}, t_{0}\right) \neq 0
\end{array}\right.
$$

and

$$
\left\{\begin{array}{c}
\phi_{t} \geq \frac{1}{n}\left(\delta_{i j}-\eta_{i} \eta_{j}\right) \phi_{x_{i} x_{j}} \text { at }\left(x_{0}, t_{0}\right) \\
\text { for some } \eta \in \mathbb{R}^{n+1} \text { with }|\eta| \neq 1, \quad \text { if } \nabla \phi\left(x_{0}, t_{0}\right)=0 .
\end{array}\right.
$$

Definition 6.3. A function $u \in C\left(\mathbb{R}^{n+1} \times[0, T)\right)$ is a weak solution of (6.5) provided $u$ is both a weak subsolution and a weak supersolution.

Lemma 6.1. Assume that $u$ is a weak subsolution in $\mathbb{R}^{n+1} \times[0, T]$ of the following equation

$$
u_{t}=\frac{1}{n}\left(\delta_{i j}-\frac{u_{x_{i}} u_{x_{j}}}{|\nabla u|^{2}}\right) u_{x_{i} x_{j}}-\theta_{1}|\nabla u|
$$

and $v$ is a weak supersolution in $\mathbb{R}^{n+1} \times[0, T]$ of the following equation

$$
v_{t}=\frac{1}{n}\left(\delta_{i j}-\frac{v_{x_{i}} v_{x_{j}}}{|\nabla u|^{2}}\right) v_{x_{i} x_{j}}-\theta_{2}|\nabla v|
$$

Suppose further

$$
\begin{aligned}
& \theta_{1} \geq \theta_{2} \\
& u \leq v \text { on } \mathbb{R}^{n+1} \times\{t=0\}
\end{aligned}
$$

Finally assume

$$
\left\{\begin{array}{r}
u \text { and } v \text { are constant, with } u \leq v \\
\quad \text { on } \mathbb{R}^{n+1} \times[0, T] \bigcap\{|x| \geq R(T)\}
\end{array}\right.
$$

for some constant $R(T) \geq 0$. Then

$$
u \leq v \text { on } \mathbb{R}^{n+1} \times[0, T] .
$$

Proof. The key technical devices in the proof are taken from [10]. For the sake of completeness, we sketch it here.

1. Should (6.12) fail, then

$$
\max _{(x, t) \in \mathbb{R}^{n+1} \times[0 . T]}(u-v) \equiv a>0 ;
$$

and so for $\alpha>0$ small enough,

$$
\max _{(x, t) \in \mathbb{R}^{n+1} \times[0, T]}(u-v-\alpha t) \geq \frac{a}{2}>0 .
$$


Define

$$
\begin{aligned}
& u^{\varepsilon}(x, t)=\sup _{y \in \mathbb{R}^{n+1}, s \in[0, T]}\left\{u(y, s)-\varepsilon^{-1}\left(|x-y|^{2}+(t-s)^{2}\right)\right\}, \\
& v_{\varepsilon}(x, t)=\inf _{y \in \mathbb{R}^{n+1}, s \in[0, T]}\left\{v(y, s)+\varepsilon^{-1}\left(|x-y|^{2}+(t-s)^{2}\right)\right\},
\end{aligned}
$$

According to (6.11) we have

$$
u^{\varepsilon}=u, v_{\varepsilon}=v, \quad \text { on }\{|x| \geq 2 R(T)\}
$$

for all small $\varepsilon>0$. Note further $u^{\varepsilon} \rightarrow u$ and $v_{\varepsilon} \rightarrow v$ uniformly by Lemma 3.1 in [10]. Consequently if we fix $\varepsilon>0$ small enough,

$$
\max _{(x, t) \in \mathbb{R}^{n+1} \times[0, T]}\left(u^{\varepsilon}-v_{\varepsilon}-\alpha t\right) \geq \frac{a}{4}>0 .
$$

2. Given $\delta>0$, define

$$
\left.\Phi(x, y, t, s)=u^{\varepsilon}(x+y, t+s)-v_{\varepsilon}(x, t)-\alpha t-\delta^{-1}\left(|y|^{4}+s^{4}\right)-\eta \frac{1}{T-t}\right] .
$$

Owing to (6.14) we see, if $\eta>0$ small enough,

$$
\max _{(x, t),(x+y, t+s) \in \mathbb{R}^{n+1} \times[0, T]} \Phi \geq \frac{a}{8}>0 .
$$

Choose now $\left(x_{1}, t_{1}\right),\left(x_{1}+y_{1}, t_{1}+s_{1}\right) \in \mathbb{R}^{n+1} \times[0, T]$ so that

$$
\Phi\left(x_{1}, y_{1}, t_{1}, s_{1}\right)=\max _{(x, t),(x+y, t+s) \in \mathbb{R}^{n+1} \times[0, T]} \Phi .
$$

Note in view of (6.13), (6.15) and Lemma 3.1 (ii) in [10] that such points exists. Since $\Phi\left(x_{1}, y_{1}, t_{1}, s_{1}\right)>0,(6.15)$ implies

$$
\left|y_{1}\right|, \quad\left|s_{1}\right| \leq C \delta^{1 / 4} .
$$

3. If $\varepsilon, \delta>0$ are fixed small enough, we have

$$
\sigma(\varepsilon)<t_{1}, t_{1}+s<T,
$$

with $\sigma(\varepsilon)$ defined in (3.3) of [10]. In fact, $\Phi \rightarrow-\infty$ as $t \rightarrow T^{-}$uniformly on $\{|x| \geq 2 R(T)\} \times\{|x| \geq 2 R(T)\}$. Hence $t_{1}, t_{1}+s<T$. The proof of $t_{1}, t_{1}+s<\sigma(\varepsilon)$ is identical that of (3.17) in [10]. Hereafter in the proof, $\alpha, \varepsilon, \delta, \eta>0$ are fixed.

According to Lemma 3.1 (vii) in [10], $u^{\varepsilon}$ is a weak subsolution near

$$
\left(x_{1}+y_{1}, t_{1}+s_{1}\right) \text {, }
$$

$v_{\varepsilon}$ is a weak supersolution near

$$
\left(x_{1}, t_{1}\right)
$$

4. Claim: $y_{1} \neq 0$.

Assume that $y_{1}=0$. Then (6.15), (6.17) imply

$$
\begin{aligned}
& u^{\varepsilon}\left(x_{1}, t_{1}+s_{1}\right)-v_{\varepsilon}\left(x_{1}, t_{1}\right)-\alpha t_{1}-\delta^{-1} s_{1}^{4}-\frac{\eta}{T-t_{1}} \\
& \quad \geq u^{\varepsilon}(x+y, t+s)-v_{\varepsilon}(x, t)-\alpha t-\delta^{-1}\left(|y|^{4}+s^{4}\right)-\frac{\eta}{T-t}
\end{aligned}
$$

for all $(x, t),(x+y, t+s) \in \mathbb{R}^{n+1} \times[0, T]$. 
First, put $x=x_{1}, t=t_{1}$ as above, and simplify to obtain

$$
u^{\varepsilon}\left(x_{1}+y, t_{1}+s\right) \leq u^{\varepsilon}\left(x_{1}, t_{1}+s_{1}\right)+\delta^{-1}\left|y^{4}\right|+\delta^{-1}\left(s^{4}-s_{1}^{4}\right)
$$

for $\left(x_{1}+y, t_{1}+s\right) \in \mathbb{R}^{n+1} \times[0, T]$. By the same computing of (3.20) in [10], we have

$$
\frac{4 s_{1}^{3}}{\delta} \leq 0
$$

Second, put $y=x_{1}-x, s=t_{1}+s_{1}-t$, then

$$
\begin{aligned}
& v_{\varepsilon}(x, t) \geq v_{\varepsilon}\left(x_{1}, t_{1}\right)+\left(\frac{4 s_{1}^{3}}{\delta}-\alpha-\frac{\eta}{\left(T-t_{1}\right)(T-t)}\right)\left(t-t_{1}\right) \\
& -6 s_{1}^{2} \frac{\left(t-t_{1}\right)^{2}}{\delta}+O\left(\left(x-x_{1}\right)^{4}+\left|t-t_{1}\right|^{4}\right) \text { as }(x, t) \rightarrow\left(x_{1}, t_{1}\right) .
\end{aligned}
$$

Note that $v_{\varepsilon}$ is a weak supersolution near $\left(x_{1}, t_{1}\right)$. By the same computing of (3.23) in [10], we have

$$
\frac{4 s_{1}^{3}}{\delta}-\alpha-\frac{\eta}{\left(T-t_{1}\right)(T-t)} \geq 0 .
$$

This yields a contradiction with $\frac{4 s_{1}^{3}}{\delta} \leq 0$.

5. By Lemma 3.1 (vi) in [10], we have

$$
(x, y, t, s) \rightarrow u^{\varepsilon}(x+y, t+s)+\varepsilon^{-1}\left(|x-y|^{2}+(t+s)^{2}\right)
$$

is convex. As

$$
(x, t) \rightarrow-v_{\varepsilon}(x, t)+\varepsilon^{-1}\left(|x|^{2}+t^{2}\right)
$$

is convex as well, we have

$$
(x, y, t, s) \rightarrow \Phi(x, y, t, s)+C\left(|x|^{2}+|y|^{2}+t^{2}+s^{2}\right)
$$

is convex near $\left(x_{1}, y_{1}, t_{1}, s_{1}\right)$, for some sufficiently large constant $C=C(\varepsilon, \delta)$. Since $\Phi$ attains its maximum at $\left(x_{1}, y_{1}, t_{1}, s_{1}\right)$, we may use Jensen theorem: there exist points $\left\{\left(x^{k}, y^{k}, t^{k}, s^{k}\right)\right\}_{k=1}^{\infty}$ such that

$$
\left(x^{k}, y^{k}, t^{k}, s^{k}\right) \rightarrow\left(x_{1}, y_{1}, t_{1}, s_{1}\right) \text { as } k \rightarrow \infty,
$$

$\Phi, u^{\varepsilon}$ and $v_{\varepsilon}$ are each twice differentiable at $\left(x^{k}, y^{k}, t^{k}, s^{k}\right)$

$$
(k=1,2, \ldots) \text {, }
$$

$$
\begin{gathered}
\nabla_{x, y, t, s} \Phi\left(x^{k}, y^{k}, t^{k}, s^{k}\right) \rightarrow 0 \text { as } k \rightarrow \infty, \\
\nabla_{x, y, t, s}^{2} \Phi\left(x^{k}, y^{k}, t^{k}, s^{k}\right) \leq o(1) I_{2(n+1)+2} \text { as } k \rightarrow \infty .
\end{gathered}
$$

6. By (6.15), (6.21), we have

$$
\begin{gathered}
\nabla_{x} \Phi\left(x^{k}, y^{k}, t^{k}, s^{k}\right)=\nabla u^{\varepsilon}\left(x^{k}+y^{k}, t^{k}+s^{k}-\nabla v_{\varepsilon}\left(x^{k}, t^{k}\right) \equiv p^{k}-\bar{p}^{k}\right. \\
\nabla_{y} \Phi\left(x^{k}, y^{k}, t^{k}, s^{k}\right) \\
=\nabla u^{\varepsilon}\left(x^{k}+y^{k}, t^{k}+s^{k}\right)-4\left|y^{k}\right|^{2} y^{k} / \delta \\
=p^{k}-4\left|y^{k}\right|^{2} y^{k} / \delta
\end{gathered}
$$


Since $y^{k} \rightarrow y_{1}$, we deduce from (6.22) that

$$
p^{k}, \bar{p}^{k} \rightarrow 4\left|y_{1}\right|^{2} y_{1} / \delta \equiv p \text { in } \mathbb{R}^{n+1}
$$

by step 4 , we have $p \neq 0$. Hence $p^{k}, \bar{p}^{k} \neq 0$ for large enough $k$.

From (6.15), (6.22) we note

$$
\begin{aligned}
\Phi_{t}\left(x^{k}, y^{k}, t^{k}, s^{k}\right) & =u_{t}^{\varepsilon}\left(x^{k}, y^{k}, t^{k}, s^{k}\right)-v_{\varepsilon t}\left(x^{k}, t^{k}\right)-\alpha-\frac{\eta}{\left(T-t^{k}\right)^{2}} \\
& \equiv q^{k}-\bar{q}^{k}-\alpha-\frac{\eta}{\left(T-t^{k}\right)^{2}} .
\end{aligned}
$$

As $u^{\varepsilon}$ and $v^{\varepsilon}$ are Lipschitz, we may assume (passing to a subsequence if necessary) that

$$
q^{k} \rightarrow q, \quad \bar{q}^{k} \rightarrow \bar{q} \quad \text { in } \mathbb{R}
$$

Then (6.22), (6.27) ensure

$$
q-\bar{q} \geq \alpha>0
$$

7. (6.15), (6.21) imply

$$
\begin{aligned}
\nabla_{x}^{2} \Phi\left(x^{k}, y^{k}, t^{k}, s^{k}\right) & =\nabla^{2} u^{\varepsilon}\left(x^{k}+y^{k}, t^{k}+s^{k}\right)-\nabla^{2} v_{\varepsilon}\left(x^{k}, t^{k}\right) \\
& \equiv R^{k}-\bar{R}^{k} .
\end{aligned}
$$

Now (6.23) forces

$$
R^{k}-\bar{R}^{k} \leq \varepsilon_{k} I_{(n+1)},
$$

where $\varepsilon_{k} \rightarrow 0$. Furthermore, by Lemma 3.1 (vi) in [10], one has $R^{k} \geq-C I_{(n+1)}$ and $\bar{R}^{k} \leq C I_{(n+1)}$, for $C=C(\varepsilon)$. Thus

$$
-C I_{(n+1)} \leq R^{k} \leq \bar{R}^{k}+\varepsilon_{k} I_{(n+1)} \leq C I_{(n+1)} .
$$

We may consequently suppose that (passing as necessary to subsequence)

$$
R^{k} \rightarrow R, \bar{R}^{k} \rightarrow \bar{R} \text { in } S^{n \times n}
$$

with

$$
R \leq \bar{R}
$$

8. Note that $p, \bar{p} \neq 0$, hence $p^{k}=\nabla u^{\varepsilon}\left(x^{k}+y^{k}, t^{k}+s^{k}\right), \bar{p}^{k}=\nabla v_{\varepsilon}\left(x^{k}, t^{k}\right)$ are nonzero for large $k$. Since $u^{\varepsilon}$ is a weak subsolution near $\left(x_{1}+y_{1}, t_{1}+s_{1}\right)$ and $v_{\varepsilon}$ is a weak supersolution near $\left(x_{1}, t_{1}\right)$, we thus have

$$
\begin{aligned}
q^{k} & \leq \frac{1}{n}\left(\delta_{i j}-p_{i}^{k} p_{j}^{k} /\left|p^{k}\right|^{2}\right) r_{i j}^{k}-\theta_{1}\left|p^{k}\right|, \\
\overline{q^{k}} & \geq \frac{1}{n}\left(\delta_{i j}-\bar{p}_{i}^{k} \bar{p}_{j}^{k} /\left|\bar{p}^{k}\right|^{2}\right) \bar{r}_{i j}^{k}-\theta_{2}\left|\bar{p}^{k}\right|
\end{aligned}
$$

for all large $k$. We send $k$ to infinity, and obtain

$$
q \leq \frac{1}{n}\left(\delta_{i j}-p_{i} p_{j} /|p|^{2}\right) r_{i j}-\theta_{1}|p|, \bar{q} \geq \frac{1}{n}\left(\delta_{i j}-p_{i} p_{j} /|p|^{2}\right) \bar{r}_{i j}-\theta_{2}|p| .
$$


Hence

$$
q-\bar{q} \leq \frac{1}{n}\left(\delta_{i j}-p_{i} p_{j} /|p|^{2}\right)\left(r_{i j}-\bar{r}_{i j}\right)-\left(\theta_{1}-\theta_{2}\right)|p| .
$$

Now the matrix $\left(\left(\delta_{i j}-p_{i} p_{j} /|P|^{2}\right)\right)$ is a nonnegative and $R-\bar{R}$ is nonpositive, $\theta_{1} \geq \theta_{2}$. Consequence $q-\bar{q} \leq 0$, a contradiction to (6.29). The proof of Lemma 6.1 is complete.

Lemma 6.2. Assume that $u_{\theta}$ is a weak solution of

$$
\left\{\begin{array}{l}
u_{t}=\frac{1}{n}\left(\delta_{i j}-u_{x_{i}} u_{x_{j}} /|\nabla u|^{2}\right) u_{x_{i} x_{j}}-\theta|\nabla u| \text { in } \mathbb{R}^{n+1} \times[0, T] \\
u(x, 0)=g(x) \text { on } \mathbb{R}^{n+1} .
\end{array}\right.
$$

Suppose that $g$ is a constant on $\{|x| \geq R(T)\}$ and $u$ is a constant on $\mathbb{R}^{n+1} \times$ $[0, T] \bigcap\{|x| \geq R(T)\}$ for some constant $R>0$. Then

$$
\lim _{\theta \rightarrow \theta_{0}}\left\|u_{\theta}-u_{\theta_{0}}\right\|_{L^{\infty}\left(\mathbb{R}^{n+1} \times[0, T]\right)}=0 .
$$

Proof. 1. Let $\theta_{n} \rightarrow \theta_{0}$ as $n \rightarrow \infty$, then there exist two numbers $\bar{\theta}, \underline{\theta}$ such that

$$
\underline{\theta} \leq \theta_{n} \leq \bar{\theta}, \quad n=1,2, \ldots
$$

Let $u_{n}, \underline{u}, \bar{u}$ be the solution of $(6.33)$ with $\theta=\theta_{n}, \underline{\theta}, \bar{\theta}$, respectively. By Lemma 6.1, we have

$$
\underline{u}(x, t) \leq u_{n}(x, t) \leq \bar{u} \text { in } \mathbb{R}^{n+1} \times[0, T] .
$$

Note that $u_{n}, \underline{u}, \bar{u}$ are constants on $\mathbb{R}^{n+1} \times[0, T] \bigcap\{|x| \geq R(T)\}$. There is a subsequence of $\left\{u_{n}\right\},\left\{u_{n_{k}}\right\}$ such that

$$
u_{n_{k}} \rightarrow v \text { uniformly on } \mathbb{R}^{n+1} \times[0, T]
$$

where $v$ is a constant on $\{|x| \geq 2 R(T)\}$.

2. Now we prove that $v$ is a weak solution of (6.33) with $\theta=\theta_{0}$.

a. Choose $\phi \in C^{2}\left(\mathbb{R}^{n+1} \times(0, T)\right)$ and suppose first $v-\phi$ has a strict local maximum at some point $\left(x_{0}, t_{0}\right) \in \mathbb{R}^{n+1} \times(0, T)$. As $u_{n_{k}} \rightarrow v$ uniformly near $\left(x_{0}, t_{0}\right), u_{n_{k}}-\phi$ has a local maximum at a point $\left(x_{k}, t_{k}\right)(k=1,2, \cdots)$ with

$$
\left(x_{k}, t_{k}\right) \rightarrow\left(x_{0}, t_{0}\right) \text { as } k \rightarrow \infty .
$$

Since $u_{k}$ is a weak solution, we have either

$$
\phi_{t} \leq \frac{1}{n}\left(\delta_{i j}-\phi_{x_{i}} \phi_{x_{j}} /|\nabla \phi|^{2}\right) \phi_{x_{i} x_{j}}-\theta|\nabla \phi| \text { at }\left(x_{k}, t_{k}\right)
$$

if $\nabla \phi\left(x_{k}, t_{k}\right) \neq 0$, or

$$
\phi_{t} \leq \frac{1}{n}\left(\delta_{i j}-\eta_{i}^{k} \eta_{j}^{k}\right) \phi_{x_{i} x_{j}} \text { at }\left(x_{k}, t_{k}\right)
$$

for some $\eta^{k} \in \mathbb{R}^{n+1}$ with $\left|\eta^{k}\right| \leq 1$, if $\nabla \phi\left(x_{k}, t_{k}\right)=0$.

b. Assume first $\nabla \phi\left(x_{0}, t_{0}\right) \neq 0$. Then $\nabla \phi\left(x_{k}, t_{k}\right) \neq 0$ for all large enough $k$. Hence we may pass to limits in the inequality (6.35) to discover

$$
\phi_{t} \leq\left(\delta_{i j}-\phi_{x_{i}} \phi_{x_{j}} /|\nabla \phi|^{2}\right) \phi_{x_{i} x_{j}}-\theta|\nabla \phi| \text { at }\left(x_{0}, t_{0}\right) \text {. }
$$

c. Suppose $\nabla \phi\left(x_{0}, t_{0}\right)=0$. We set

$$
\xi^{k}= \begin{cases}(\nabla \phi /|\nabla \phi|)\left(x_{k}, t_{k}\right) & \text { if } \nabla \phi\left(x_{k}, t_{k}\right) \neq 0 \\ \eta^{k} & \text { if } \nabla \phi\left(x_{k}, t_{k}\right)=0\end{cases}
$$


Passing if necessary to a subsequence we may assume $\xi^{k} \rightarrow \eta$. Then $|\eta| \leq 1$. Utilizing (6.36), we deduce as well

$$
\phi_{t} \leq \frac{1}{n}\left(\delta_{i j}-\eta_{i} \eta_{j}\right) \phi_{x_{i} x_{j}} \text { at }\left(x_{0}, t_{0}\right) .
$$

d. If $v-\phi$ has only a local maximum at $\left(x_{0}, t_{0}\right)$, we apply the above argument to

$$
\psi(x, t) \equiv \phi(x, t)+\left|x-x_{0}\right|^{4}+\left(t-t_{0}\right)^{4},
$$

so that $v-\psi$ has a strict local maximum at $\left(x_{0}, t_{0}\right)$. Hence $u$ is a weak subsolution of (6.33) with $\theta=\theta_{0}$. Similar reasoning verifies that $v$ is a weak supersolution of (6.33) with $\theta=\theta_{0}$. Hence $v=u_{\theta_{0}}$.

3. $\lim _{\theta \rightarrow \theta_{0}}\left\|u_{\theta}-u_{\theta_{0}}\right\|_{L^{\infty}\left(\mathbb{R}^{n+1} \times[0, T]\right)}=0$.

If this conclusion is fail, then there exist a positive number $\varepsilon_{0}>0$ and a sequence $\left\{\theta_{n}\right\}$ such that

$$
\theta_{n} \rightarrow \theta_{0} \text { as } n \rightarrow \infty
$$

but

$$
\left\|u_{\theta_{n}}-u_{\theta_{0}}\right\|_{L^{\infty}\left(\mathbb{R}^{n+1} \times[0, T]\right)} \geq \varepsilon_{0}>0
$$

By step 1,2, there exists a subsequence $\left\{u_{\theta_{n_{k}}}\right\}$ of $\left\{u_{\theta_{n}}\right\}$ such that

$$
u_{\theta_{n_{k}}} \rightarrow u_{\theta_{0}} \text { uniformly on } \mathbb{R}^{n+1} \times[0, T],
$$

as $k \rightarrow \infty$. This yields a contradiction with (6.37). The proof of Lemma 6.4 is completed.

The proof of Proposition 6.2. Let $U_{0}$ be a smooth domain bounded by $M_{0}$. Choose a continuous function $g: \mathbb{R}^{n+1} \rightarrow R$ satisfying

$$
M_{0}=\left\{x \in \mathbb{R}^{n+1}: g(x)=0\right\},
$$

$g$ is a constant on $\mathbb{R}^{n+1} \bigcap\{|x| \geq s\}$ for some $s>0$, and $U_{0}=\{x: g(x)<0\}$.

Let $u, u_{1}$ be weak solution of (6.33) with $\theta=\theta, \theta_{1}$ respectively.

Then, note that a classical solution is a weak solution (see [10]), we have

$$
\begin{aligned}
& M_{t}=\{x: u(x, t)=0\}, \quad 0 \leq t \leq t_{\max }, \\
& M_{t}^{1}=\left\{x: u_{1}(x, t)=0\right\}, \quad 0 \leq t \leq t_{1 \max }, \\
& U_{t}=\{x: u(x, t)<0\}, \\
& U_{t}^{1}=\left\{x: u_{1}(x, t)<0\right\} .
\end{aligned}
$$

By Lemma 6.3, we have

$$
u(x, t) \leq v(x, t) \text { on } \mathbb{R}^{n+1} \times[0, T] .
$$

Hence

$$
U_{t} \subset U_{t}^{1}
$$

The proof of Proposition 6.2 is completed.

Lemma 6.3. Assume $M_{0}$ is a compact, uniformly convex n-dimensional hypersurface without boundary. Let $F$ be a solution of problem (1.2)-(1.3) with positive $\theta$. Denotes the mean curvature of $M_{0}$ by $H_{0}$. Then, we have

(a) If $\min _{M_{0}} H_{0}>\theta$, then $t_{\max }$ is finite and $M_{t}$ shrinks to point. 
(b) If $\max _{M_{0}} H_{0}<\theta$, then $t_{\max }=\infty$, and the domain $U_{t}$ bounded by $M_{t}$ occupies the entire space as $t$ approaches infinity.

Proof. (a) Define $v=H-\theta$, then

$$
\left\{\begin{array}{l}
\frac{\partial v}{\partial t}=\Delta v+|A|^{2} v \\
v(x, 0)>0
\end{array}\right.
$$

So, by the maximum principle, we have

$$
v(x, t) \geq 0, \quad \forall t>0 .
$$

Let $v_{\min }(t)=\min _{x \in M_{t}}(v-\theta)$, then

$$
\frac{d v_{\min }}{d t} \geq 0
$$

Hence

$$
v(x, t) \geq v_{\min }(t) \geq v_{\min }(0)>0 .
$$

So $H(x, t)$ has a uniform positive lower bound. Combining this with Lemma 2.2 (i), one yields

$$
\frac{d v_{\min }}{d t} \geq C_{10} v_{\min }, \text { for some } C_{10}>0 .
$$

If $t_{\max }=\infty, v_{\min }$ grows at least exponentially. From Lemma 2.2 (i), we have

$$
\frac{d v_{\min }}{d t} \geq C_{11} v_{\min }^{3}, \text { for some } C_{11}>0,
$$

for large $t$. Hence $v_{\text {min }}$ blows up in finite time, and the contradiction holds. So $t_{\max }$ must be finite.

(b) Let $v_{\max }(t)=\max _{M_{t}}(H-\theta)$, one can see that $v_{\max }(t)$ is nonincreasing. In particular,

$$
v_{\max }(t) \leq v_{\max }(0)<0
$$

Hence

$$
0 \leq H(x, t) \leq \theta, \quad \forall x, \forall t \geq 0
$$

By Theorem 5.6, $t_{\max }=\infty$. Define

$$
d(x, t)=\operatorname{dist}\left(x, M_{t}\right), \quad x \in U_{t} .
$$

Note that $M_{t}$ is convex, there exists a unique point $y \in M_{t}$ verifying $d(x, t)=$ $|x-y|$. Then

$$
d_{t}(x, t)=(\operatorname{div} \nu)(y, t)+\theta
$$

where $\nu=\nabla d$ is unit normal vector of $M_{t}$ at $(y, t)$.

Let $k_{1}, \cdots, k_{n}$ be the principle curvatures of $M_{t}$ at the point $y$, calculated with respect to the unit normal field $\nu$. Thus $(\operatorname{div} \nu)(y, t)=-\left(k_{1}+\cdots+k_{n}\right)$, and we have

$$
d_{t}=-v \geq-v_{\max }(0)>0,
$$

that is

$$
d(x, t) \geq-v_{\max }(0) / t+d(x, 0)
$$


Thus $M_{t}$ encloses any bounded set for sufficiently large $t$.

By Proposition 6.1, 6.2 and Lemma 6.3, we have the following

Lemma 6.4. Assume $M_{0}$ is a compact, uniformly convex n-dimensional hypersurface without boundary. Let $M_{t}$ be the solution of (1.2), (1.3) and $\theta>0$.

(a) If $M_{0}$ is contained inside a ball of radius less than $\frac{1}{\theta}$, then $M_{t}$ shrinks to a point and $t_{\max }$ is finite.

(b) If $M_{0}$ contains a ball of radius greater than $\frac{1}{\theta}$, then $M_{t}$ expands to infinity.

Define $I_{1}=\left\{\theta: M_{t}\right.$ is a solution of $(6.1)_{\theta},(6.2)$ that shrinks to a point $\}$, and $I_{2}=\left\{\theta: M_{t}\right.$ is a solution of $(6.1)_{\theta},(6.2)$ that expands to infinity as $t \rightarrow \infty\}$.

By Lemma 6.4 (a), we know that $t_{\max }$ is finite if $\theta \in I_{1}$. By the continuous dependence of $F$ on $\theta$, both $I_{1}$ and $I_{2}$ are open. By Lemma 6.4, they are also nonempty.

Lemma 6.5. $I_{1}$ and $I_{2}$ are open.

Proof. 1. $I_{1}$ is open.

Let $\theta_{0} \in I_{1}$. Choose a continuous function $g: \mathbb{R}^{n+1} \rightarrow \mathbb{R}$ satisfying

$$
\begin{array}{r}
M_{0}=\left\{x \in \mathbb{R}^{n+1}, g(x)=0\right\}, \\
g \text { constant on } \mathbb{R}^{n+1} \bigcap\{|x| \geq s\}
\end{array}
$$

for some $s>0$, and $U_{0}=\{x \mid g(x)<0\}$, where $U_{0}$ is a domain bounded by $M_{0}$. Let $u$ be a unique weak solution of

$$
\left\{\begin{array}{l}
u_{t}=\frac{1}{n}\left(\delta_{i j}-u_{x_{i}} u_{x_{j}} /|\nabla u|^{2}\right) u_{x_{i} x_{j}}-\theta_{0}|\nabla u| \text { in } \mathbb{R}^{n+1} \times(0, T), \\
u(x, 0)=g \text { on } \mathbb{R}^{n+1}
\end{array}\right.
$$

and $v$ be a unique weak solution of

$$
\left\{\begin{array}{l}
v_{t}=\frac{1}{n}\left(\delta_{i j}-v_{x_{i}} v_{x_{j}} /|\nabla v|^{2}\right) v_{x_{i} x_{j}}-\theta|\nabla v| \text { in } \mathbb{R}^{n+1} \times(0, T), \\
v(x, 0)=g \text { on } \mathbb{R}^{n+1}
\end{array}\right.
$$

then $M_{t}=\{x: u(x, t)=0\}\left(0 \leq t \leq t_{\max }\right)$ is the solution of the following equation

$$
\left\{\begin{array}{l}
\frac{\partial F}{\partial t}=-H+\theta_{0} \\
F(\cdot, 0)=F_{0}
\end{array}\right.
$$

$M_{t}^{\prime}=\{x: v(x, t)=0\}\left(0 \leq t \leq t_{\max }^{\prime}\right)$ is the solution of the equation

$$
\left\{\begin{array}{l}
\frac{\partial F^{\prime}}{\partial t}=-H^{\prime}+\theta \\
F(\cdot, 0)=F_{0}
\end{array}\right.
$$

Denote

$$
\begin{aligned}
& U_{t}=\left\{x \in \mathbb{R}^{n+1}: u(x, t)<0\right\}, \\
& U_{t}^{\prime}=\left\{x \in \mathbb{R}^{n+1}: v(x, t)<0\right\} .
\end{aligned}
$$

Since $M_{t}$ tends to a point $p$ as $t \rightarrow t_{\max }$, there exists a $T_{0}>0$ such that

$$
U_{t} \subset \subset B_{1 / 2 \theta}(p), \quad \forall t \geq T_{0},
$$


where $B_{1 / 2 \theta}(p)$ is a ball of radius $1 / 2 \theta$. Hence, $\forall t \geq T_{0}$,

$$
U(x, t) \geq \alpha_{0}>0, \text { in } \mathbb{R}^{n+1} \backslash B_{1 / \theta}(p) .
$$

By Lemma 6.2 , if $\left|\theta-\theta_{0}\right|<\varepsilon$, then, $\forall t \geq T_{0}$,

$$
v(x, t) \geq \frac{1}{2} \alpha_{0} \text { in } \mathbb{R}^{n+1} \backslash B_{1 / \theta}(p) .
$$

Hence

$$
U_{t}^{\prime} \subset B_{1 / \theta}(p), \quad \forall t \geq T_{0} .
$$

By Lemma 6.4 (a), $M_{t}^{\prime}$ shrinks to a point as $t \rightarrow t_{\max }^{\prime}$. Thus $\left(\theta_{0}-\frac{1}{2} \alpha_{0}, \theta_{0}+\right.$ $\left.\frac{1}{2} \alpha\right) \subset I_{1}$. So $I_{1}$ is a open set.

2. Similarly, $I_{2}$ is a open set.

Using comparison principle Proposition 6.1, Proposition 6.2, we conclude $I_{1}=(0, \underline{\theta})$ and $I_{2}=(\bar{\theta},+\infty)$, where $0 \leq \underline{\theta} \leq \bar{\theta}$. From Theorem 5.6, we know that for each $\theta \in[\underline{\theta}, \bar{\theta}]$, the flow $(6.1)_{\theta}$, and (6.2) exists for all time.

Lemma 6.6. Assume $M_{0}$ is a compact, uniformly convex $n$ dimensional hypersurface without boundary. Let $F$ satisfy $(6.1)_{\theta}$ and (6.2) for $\theta \in[\underline{\theta}, \bar{\theta}]$. Then $H(\cdot, t)$ is uniformly bounded in $[0, \infty)$.

Proof. Suppose there is a sequence of times $T_{i} \rightarrow+\infty$ such that

$$
\max _{M_{T_{i}}} H=H_{T_{i}} \quad \text { and } \quad H_{T_{i}} \rightarrow \infty \text { as } i \rightarrow \infty .
$$

Then for any $\eta>0$ there is $i$ such that for all $j \geq i$,

$$
|\nabla H| \leq \frac{1}{2} \eta H_{\max }^{2} \text { on } M_{T_{j}} .
$$

We can then use Theorem 4.1 and Meyer's theorem as in [16] to conclude that

$$
H_{\min } \geq(1-\eta) H_{\max } \text { on } M_{T_{j}} .
$$

Theorem 3.1 then implies that all principal curvatures on $M_{T_{j}}$ tend to infinity which is clearly a contradiction since the enclosed volume is large than some positive number.

Now we begin to study the asymptotic behavior of $M_{t}$ as $t \rightarrow \infty$ for $\theta \in[\underline{\theta}, \bar{\theta}]$. Evolution equation (2.3) gives

$$
\left(\frac{\partial}{\partial t}-\triangle\right)\left\{e^{\delta t}(H-\theta)^{2}-K\right\} \leq-e^{\delta t}(H-\theta)^{2}|A|^{2}+\delta e^{\delta t}(H-\theta)^{2}
$$

for any constants $\delta>0$ and $K \in \mathbb{R}$. Choose $\delta=\inf _{M_{t}}|A|^{2}$ (which is strictly greater than zero by Theorem 2.3) and $K=\sup _{M_{0}}\left\{(H-\theta)^{2}\right\}$ (which is bounded by assumption). Then $\left.\left\{e^{\delta t}(H-\theta)^{2}-K\right\}\right|_{t=0} \leq 0$ and

$$
\left(\frac{\partial}{\partial t}-\triangle\right)\left\{e^{\delta t}(H-\theta)^{2}-K\right\} \leq 0 .
$$

The maximum principle yields

$$
(H-\theta)^{2} \leq K e^{-\delta t}
$$


for all $t>0$ which imply that $\sup _{M_{t}}|H-\theta|$ tends to zero as $t \rightarrow+\infty$. Thus $H$ is bounded from below by $\frac{\theta}{2}$ for $t \geq T_{1}$.

Hence, from Lemma 3.2 we see that

$$
f_{0}=\left(|A|^{2}-\frac{1}{n} H^{2}\right) / H^{2}
$$

satisfies the inequality

$$
\frac{\partial}{\partial t} f_{0} \leq \Delta f_{0}+\frac{2}{H}\left\langle\nabla_{l} H, \nabla_{l} f_{0}\right\rangle-\varepsilon^{2} \theta^{2} f_{0}
$$

This shows

$$
|A|^{2}-\frac{1}{n} H^{2} \leq C e^{-\delta_{1} t},
$$

and a slight modification in the Proof of Theorem 5.1 shows that all higher derivatives of the second functional form decay exponentially as well. In particular

$$
\left|\frac{d}{d t} F\right|=|H-\theta| \rightarrow 0 \text { as } t \rightarrow \infty .
$$

Thus $M_{t}$ converges to a limiting hypersurface $M_{\infty}^{\theta}$, and in view of $(6.1)$ the limiting hypersurface $M_{\infty}^{\theta}$ is a sphere with radius $1 / \theta$. We have

Lemma 6.7. Assume $M_{0}$ is a compact, uniformly convex n-dimensional hypersurface without boundary. Let $M_{t}$ be the solution of (6.1) ${ }_{\theta}$ and (6.2), $\theta \in[\underline{\theta}, \bar{\theta}]$. Then $M_{t} \rightarrow M_{\infty}^{\theta}$ as $t \rightarrow \infty$.

Proposition 6.3. For every uniformly convex initial surface $M_{0}$, there exists $\theta^{*}>0$ such that the following hold.

(a) The solution $M_{t}$ of $(6.1)_{\theta}$ and (6.2) shrinks to a point in finite time when $0<\theta<\theta^{*}$.

(b) The solution of $(6.1)_{\theta^{*}}$ and (6.2), converges to a sphere with radius $1 / \theta^{*}$.

(c) The solution of $(6.1)_{\theta}$ and (6.2) expands to infinity as $t \rightarrow \infty$ when $\theta>\theta^{*}$.

Proof. We only need to show that $\underline{\theta}=\bar{\theta}$. Let $\underline{M}_{t}$ and $\bar{M}_{t}$ be the solution of $(6.1)_{\theta}$ and $(6.2)$ for $\theta=\underline{\theta}$ and $\bar{\theta}$, respectively.

On one hand, by Proposition 6.2, we have

$$
\underline{M}_{t} \subset \bar{U}_{t}, \quad t \geq 0 \text {. }
$$

However, on the other hand, let

$$
d(x, t)=\left\{\begin{aligned}
-\operatorname{dist}\left(x, \underline{M}_{t}\right) & \text { if } x \in \underline{U}_{t} \\
\operatorname{dist}\left(x, \underline{M}_{t}\right) & \text { if } x \in \mathbb{R}^{n+1} \backslash \underline{U}_{t},
\end{aligned}\right.
$$

then $d(x, t)$ is smooth in

$$
Q=\left\{(x, t):-\delta_{0} \leq \underline{d}(x, t) \leq \delta_{0}, 0 \leq t \leq t_{0}\right\},
$$

where $\delta_{0}$ and $t_{0}$ are small enough, $U_{t}$ is the bounded region enveloped by $\underline{M}_{t}$. As [11], we have

$$
\underline{d}_{t}=F\left(\nabla^{2} \underline{d}, \underline{d}\right)+\underline{\theta} \text { in } Q .
$$


Similarly the signed distance

$$
\bar{d}_{t}(x, t)=\left\{\begin{aligned}
-\operatorname{dist}\left(x, \bar{M}_{t}\right) & \text { if } x \in \bar{U}_{t} \\
\operatorname{dist}\left(x, \bar{M}_{t}\right) & \text { if } x \in \mathbb{R}^{n+1} \backslash \bar{U}_{t}
\end{aligned}\right.
$$

is smooth in $Q$, provided $\delta_{0}, t_{0}>0$ are sufficiently small. Also

$$
\bar{d}_{t}=F\left(\nabla^{2} \bar{d}, \bar{d}\right)+\bar{\theta} \text { in } Q .
$$

Set

$$
w=\bar{d}-\underline{d} .
$$

From (6.40), (6.41) it follows that

$$
\left\{\begin{array}{l}
w_{t}=a_{i j} w_{x_{i} x_{j}}+c w+(\bar{\theta}-\underline{\theta}), \\
w(x, 0)=0
\end{array}\right.
$$

for

$$
\begin{aligned}
& a_{i j}=\int_{0}^{1} \frac{\partial F}{\partial r_{i j}}\left(t \nabla^{2} \bar{d}+(1-t) \nabla^{2} \underline{d}, t \bar{d}+(1-t) \underline{d}\right) d t, \\
& c=\int_{0}^{1} \frac{\partial F}{\partial z}\left(t \nabla^{2} \bar{d}+(1-t) \nabla^{2} \underline{d}, t \bar{d}+(1-t) \underline{d}\right) d t
\end{aligned}
$$

where $F$ is defined by $(2.3)$ in [11].

According to Lemma 2.1 in [11] the coefficients $\left(\left(a_{i j}\right)\right)$ are uniformly positive definite if $\delta_{0}, t_{0}$ are small enough. Also, one has that $c \geq 0$ is a bounded function in $\bar{Q}$. If $\bar{\theta}>\underline{\theta}$, set $v(t)=\min _{Q \cap\{t=t\}} w(x, t)$, then $\min _{Q \cap\{t=t\}}(\bar{d}-\underline{d})$ is increasing and becomes positive for sufficiently small $t>0$. Hence

$$
\bar{d}(x, t)>\underline{d}(x, t) \text { for } 0<t \leq t_{0}
$$

if $t_{0}$ is small enough, contradicting $\underline{M}_{t} \subset \bar{U}_{t}$ for $t \geq 0$. So we must have $\bar{\theta}=\underline{\theta}$. The proof of proposition 6.3 is completed.

\section{Convergence to a sphere}

According to Proposition 6.3, the solution $M_{t}$ of $(6.1)_{\theta}, \theta>\theta^{*}$, and (6.2) possesses the following property: For any bounded subset $K$ of $\mathbb{R}^{n+1}$, there exists $t_{K}$ such that $K$ is contained inside $M_{t}$ for all $t \geq t_{K}$. In this section, we study the asymptotic behavior of this solution.

Intuitively in this case, the mean curvature of $M_{t}$ is eventually negligible. In view of (1.2), the volume of $U_{t}$ grows linearly. Therefore, we consider the normalization given by

$$
\tilde{F}(\cdot, t)=F(\cdot, t) / t
$$

Lemma 7.1. There exists a $K_{0}$ that depends on $\theta$ and $M_{0}$ such that $\tilde{H}$ is uniformly bounded if

$$
\max _{x \in M_{0}} H(x, 0) \leq K_{0}
$$

where $\tilde{H}$ is the mean curvature of $\tilde{M}_{t}=M_{t} / t$. 
Proof. From Lemma 2.2 (i), we have

$$
\frac{\partial}{\partial t} H=\Delta H+(H-\theta)|A|^{2} .
$$

Thus

$$
H \leq K_{0}, \quad x \in M_{t}, t \geq 0 .
$$

Hence, if $H-\theta \leq K_{0}-\theta<0$, we have

$$
\begin{aligned}
\frac{\partial}{\partial t} H & =\Delta H+(H-\theta)|A|^{2} \\
& \leq \Delta H+\left(K_{0}-\theta\right) \frac{1}{n}|H|^{2} \\
& :=\Delta H-v_{0} H^{2} .
\end{aligned}
$$

So

$$
\frac{\partial}{\partial t} H_{\max } \leq-v_{0} H_{\max }^{2}(t)
$$

This implies

$$
\begin{gathered}
H_{\max }(t) \leq \frac{H_{\max }(t)}{1+v_{0}+H_{\max }(t) t}, \quad t \in[0, \infty), \\
\tilde{H}_{\max }(t)=t H_{\max }(t) \leq \frac{1}{v_{0}}, \quad t \in[0, \infty) .
\end{gathered}
$$

Lemma 7.2. Let $\tilde{m}_{c}$ be the support function of $\tilde{M}_{t}$ for $(6.1)_{\theta}, \theta>0$, of which the initial surface is a large sphere which center is origin with radius $R_{0}$. Then

$$
\tilde{m}_{c}(\cdot, t)-\theta \rightarrow 0
$$

uniformly as $t \rightarrow \infty$.

Proof. Let $R(t)=(\tilde{F}, \tilde{\nu})$, where $\tilde{\nu}$ is the unit normal vector of $\tilde{M}_{t}$. Then

$$
\frac{d R}{d t}=-\frac{1}{t} R-\frac{1}{2 t^{2} R}+\frac{\theta}{t} .
$$

Set $\tau=\log t$, we have

$$
\frac{d R}{d \tau}=-R-e^{-\tau} \frac{1}{2 R}+\theta
$$

Hence

$$
\frac{d}{d t}\left[e^{\tau} \cdot(R-\theta)\right]=-\frac{1}{2 R} .
$$

So

$$
e^{\tau}(R(\tau)-\theta)=R(0)-\theta-\int_{0}^{\tau} \frac{1}{2 R(t)} d t
$$




$$
\begin{aligned}
|R(\tau)-\theta| & =\left|(R(0)-\theta) e^{-\tau}-e^{-\tau} \int_{0}^{\tau} \frac{d t}{2 R(t)}\right| \\
& \leq(R(0)-\theta) e^{-\tau}+e^{-\tau} \int_{0}^{\tau} \frac{d t}{2 R(t)} \\
& \leq M \tau e^{-\tau} \quad \text { if } R(0) \geq 2 \theta
\end{aligned}
$$

where $M=R(0)-\theta+v_{0}$. This proves the Lemma 7.2.

Theorem 7.3. $\tilde{m}(\cdot, \tau)-\theta$ tends to zero uniformly as $\tau \rightarrow \infty$.

Proof. Since $M_{t}$ expands, we may assume, without loss of generality, that $M_{t}$ is pinched between two large circle. Then the desired result follows from Lemma 7.2.

\section{Acknowledgements}

The author acknowledges the hospitality of The Institute of Mathematical Sciences at Chinese University of Hong Kong where parts of this work was carried out. This work is supported by the National Natural Science Foundation of China (No.11071206).

\section{References}

[1] Abresch, U., Langer, J.: The normalized curve shortening flow and homothetic solutions. J. Differ. Geom. 23, 175-196 (1986)

[2] Altschuler, S., Angenent, S.B., Giga, Y.: Mean curvature flow through singularities for surfaces of rotation. J. Geom. Anal. 5, 293-358 (1995)

[3] Angenent, S.B., Velazquez, J.J.L.: Degenerate neckpinches in mean curvature flow. J. Reine Angew. Math. 482, 15-66 (1997)

[4] Almgren, F., Taylor, J.E., Wang, L.: Curvature-driven flows: a variational approach. SIAM J. Control Optim. 31, 387-437 (1993)

[5] Almgren, F., Wang, L.: Mathematical existence of crystal growth with GibbsThomson curvature effects. Preprint (1994)

[6] Brakke, S.B.: The motion of a surface by its mean curvature. Math. Notes. Princeton University Press, Princeton (1978)

[7] Chen, Y.G., Giga, Y., Goto, S.: Uniqueness and existence of viscosity solutions of generalized mean curvature flow equations. J. Differ. Geom. 33, 749-786 (1991)

[8] Chen, X., Reitich, F.: The local existence and uniqueness of classical solutions of the Stefan problem with surface tension and kinetic undercooling. J. Math. Anal. Appl. 164, 350-362 (1992)

[9] Chou, K.S., Zhu, X.P.: Anisotropic flows for convex plane curves. Duke Math. J. 97, 579-619 (1999) 
[10] Evans, L.C., Spruck, J.: Motion of level sets by mean curvature, I. J. Differ. Geom. 33, 635-681 (1991)

[11] Evans, L.C., Spruck, J.: of level sets by mean curvature, II. Trans. Am. Math. Soc. 330, 321-332 (1992)

[12] Gage, M., Hamilton, R.S.: The shrinking of convex plane curves by the heat equation. J. Differ. Geom. 23, 69-96 (1986)

[13] Grayson, M.A.: The heat equation shrinks embedded plane curves into points. J. Differ. Geom. 26, 285-314 (1987)

[14] Gurtin, M.: Multiphase thermomechanics with interfacial structure. I. Heat conduction and the capillary balance law. Arch. Ration. Mech. Anal. 104, 195-221 (1988)

[15] Hamilton, R.S.: Three-manifolds with positive Ricci curvature. J. Differ. Geom. 17, 255-306 (1982)

[16] Huisken, G.: Flow by mean curvature of convex surfaces into spheres. J. Differ. Geom. 20, 237-266 (1984)

[17] Huisken, G.: Asymptotic behavior for singularities of the mean curvature flow. J. Differ. Geom. 31, 285-299 (1990)

[18] Huisken, G., Carlo, S.: Mean curvature flow singularities for mean convex surfaces. Calc. Var. Partial Differ. Equ. 8, 1-14 (1999)

[19] Huisken, G.: Asymptotic behavior for singularities of the mean curvature flow. J. Differ. Geom. 31, 285-299 (1990)

[20] Huisken, G.: Local and global behaviour of hypersurfaces moving by mean curvature. Proc. Symp. Pure Math. 54, 175-191 (1993)

[21] Ilmanen, T.: Elliptic regularization and partial regularity for motion by mean curvature. Mem. Am. Math. Soc. 108 (1994)

[22] Ilmanen, T.: Generalized flow of sets by mean curvature on a manifold. Indiana Univ. Math. J. 41(3), 671-705 (1992)

[23] Luckhaus, S., Sturzenhecker, T.: Implicit time discretization for the mean curvature flow equation. Calc. Var. Partial Differ. Equ. 3, 253-271 (1995)

[24] Visintin, A.: Nucleation and mean curvature flow. Commun. Partial Differ. Equ. 23, 17-53 (1998)

[25] Visintin, A.: Models of Phase Transition. Birkhäuser, Boston (1996)

[26] Smoczyk, K.: Starshaped hypersurfaces and the mean curvature flow. Manuscr. Math. 95, 225-236 (1998)

[27] Soner, M.H.: Motion of a set by the curvature of its boundary. J. Differ. Equ. 101, 313-372 (1993) 
[28] White, B.: The size of the singular set in mean curvature flow of mean-convex sets. J. Am. Math. Soc. 13, 665-695 (2000)

[29] White, B.: The nature of singularities in mean curvature flow of mean-convex sets. J. Am. Math. Soc. 16, 123-138 (2003)

[30] White, B.: A local regularity theorem for mean curvature flow. Ann. Math. (2) 161, 1487-1519 (2005)

Zuhan Liu

Department of Mathematics

Xuzhou Normal University

Xuzhou 221116

China

e-mail: zuhanl@yahoo.com

\section{Zuhan Liu}

Department of Mathematics

Yangzhou University

Yangzhou 225002

China

Received: 11 July 2011.

Accepted: 28 April 2012. 